TITLE:

\title{
An Asymptotic Comparison of Differentiable Dynamics and Tropical Geometry
}

AUTHOR(S):

Kato, Tsuyoshi

\section{CITATION:}

Kato, Tsuyoshi. An Asymptotic Comparison of Differentiable Dynamics and Tropical Geometry. Mathematical Physics, Analysis and Geometry 2011, 14(1): 39-82

\section{ISSUE DATE:}

2011-03

URL:

http://hdl.handle.net/2433/174157

\section{RIGHT:}

The final publication is available at link.springer.com; この論文は出版 社版でありません。引用の際には出版社版をご確認ご利用ください。; This is not the published version. Please cite only the published version. 


\title{
An asymptotic comparison of differentiable dynamics and tropical geometry
}

\author{
Tsuyoshi Kato
}

\begin{abstract}
In this paper we introduce a new comparison method to give rough asymptotic estimates of different evolutional dynamics. It uses a kind of scale transform called tropical geometry, which connects automata with real rational dynamics. By this procedure the defining equations are transformed rather than solutions themselves. Real rational dynamics is regarded as an approximation of evolutional dynamics given by partial differential equations. Two different evolutional dynamics can be considered when their defining equations are transformed to the same automata at infinity.
\end{abstract}

\section{Introduction}

1.A Asymptotic comparison between solutions to different PDEs: Scaling limits connect several dynamics whose features are often very different mutually. One of particular properties of scaling limits is that in many cases such associations are not injective. When two dynamical systems correspond to the same one by such scaling limits, then one might say that these two dynamics behave by the same way at infinity, and so expect that they will hold some common structural similarity.

Motivated by such aspects, in this paper we study large scale analytic properties of solutions to evolutional differential equations by use of a particular type of scaling limit. It consists of two steps, where one is to associate discrete dynamics given by real rational functions from differential equations, and the second is automata given by $(\max ,+)$-functions from the rational 
dynamics, which appears in tropical geometry. Combination of these two steps gives a process of association of automata from PDE. As above one of the important observations for the process is that it is not one to one, and so different differential equations can correspond to the same dynamics by automata. The situation can be interpreted that large (also very small) valued solutions to these PDEs admit mutual analytic relations in some sense, which we would expect to lead us to large scale analysis of structure for classes of differential equations.

In this paper we introduce a new method for study of solutions of non linear partial differential equations. Our main interest here is to obtain relative estimates of asymptotic growth of solutions to different PDEs with respect to higher derivatives and initial conditions.

For $T_{0} \in(0, \infty]$, let $u:(0, \infty) \times\left[0, T_{0}\right) \rightarrow(0, \infty)$ be a function of class $C^{\alpha+1}$. Then we introduce uniform norm of $u$ of order $\alpha+1$ by:

$$
\|u\|_{\alpha+1}=\max _{\partial_{i}=\partial_{x}, \partial_{s}}\left\{\left\|\frac{\partial^{\alpha+1} u}{\partial_{1} \ldots \partial_{\alpha+1}}\right\|_{C^{0}\left((0, \infty) \times\left[0, T_{0}\right)\right)}\right\} .
$$

Let $c=\inf _{(x, s) \in(0, \infty) \times\left[0, T_{0}\right)} u(x, s) \geq 0$ be the infimum of $u$. Suppose $u(x, s) \geq c>0$ is positive. Then we introduce the higher derivative rates by:

$$
K(u) \equiv \frac{\|u\|_{\alpha+1}}{c}
$$

and call them the derivative rates of order $\alpha+1$. Notice that even when $c$ is sufficiently large, still $K$ can be small when functions $u$ are 'near' polynomial of order less than $\alpha$.

Let $u, v:(0, \infty) \times\left[0, T_{0}\right) \rightarrow(0, \infty)$ be two functions of class $C^{\alpha+1}$. For small $\epsilon>0$, we introduce the initial rates:

$$
[u: v]_{\epsilon} \equiv \sup _{(x, s) \in(0, \infty) \times\left[0, \epsilon^{q}\right] \cup\left(0, \epsilon^{p}\right] \times\left[0, T_{0}\right)}\left(\frac{u(x, s)}{v(x, s)}\right)^{ \pm 1} .
$$

Our method provides with asymptotic relative growth very explicitly for solutions to different differential equations, with respect to their higher derivative rates and initial rates. Let us consider two differential equations of order at most $\alpha, P\left(u, u_{x}, u_{s}, u_{2 x}, u_{x s}, \ldots\right)=0$ and $Q\left(v, v_{x}, v_{s}, v_{2 x}, v_{x s}, \ldots\right)=$ 0 , and take positive solutions $u, v:(0, \infty) \times\left[0, T_{0}\right) \rightarrow(0, \infty)$ of class $C^{\alpha+1}$. When both $P$ and $Q$ are 'induced from the same automaton $\varphi$ ' which we clarify below, then we verify that there exist constants $C=C(y, r, K)$ which 
depend only on the 'rough structure' of the differential equations $P$ and $Q$, which are independent of individual solutions, so that they satisfy uniform bounds:

$$
\frac{u(x, s)}{v(x, s)}, \frac{v(x, s)}{u(x, s)} \leq C(x+k s, r, K)
$$

when their higher derivative rates and intial rates satisfy bounds $K(u), K(v) \leq$ $K$ and $[u: v]_{(L+1)(2 C K)^{-1}} \leq r$ respectively. Here $k, L=\max (l, d)$ and $C$ are explicit constants which arise from scaling limits of these PDE as below.

Let us pick up the required information to determine the constants $C(y, r, K)$. Our basic process is to extract very rough framework of structures of PDE. They are given by $n$ variable rational dynamics of the form:

$$
z_{N+1}^{t+1}=f\left(z_{N-l_{0}}^{t+1}, \ldots, z_{N}^{t+1}, z_{N-l_{1}}^{t}, \ldots, z_{N+k_{1}}^{t}, \ldots, z_{N+k_{d+1}}^{t-d}\right)
$$

and scaling parameters $z_{N}^{t}=\epsilon^{m} u(x, s)$ and $(N, t)=\left(\epsilon^{-p} x, \epsilon^{-q} s\right)$.

Once such reductions are given, then automata $\varphi$ are canonically associated, and at this stage, one has chosen several numbers $L=\max (l, d), k, n$, $D=\max (p, q)$ and $C$, where $l=\max \left(l_{0}, \ldots, l_{d+1}\right), k=\max \left(k_{1}, \ldots, k_{d+1}\right)$, and $C$ are the coefficients of $\alpha+1$ derivatives in the Taylor expansions, called error constants (3.B.2.2). Relative $(\max ,+)$-functions $\varphi$ are piecewise linear and they are Lipschitz. So one obtains particular two data $M$ and $c$, where $M$ is the number of the components $(1 . B)$ and $c$ is the Lipschitz constants both for $\varphi$. In total at the level of defining equations of dynamics, induction of rational functions and scaling parameters determine the above seven data. In section 4 we see that these constants are explicitly calculated or estimated in concrete cases.

On the other hand individual solutions give the constants $[u: v]_{\epsilon}$ and $K$, Now $C(y, r, K)$ are in fact given quite explicitly as below. The above numbers are all the data which we need for the above asymptotic estimates among applicable pairs of PDEs.

As a general procedure, the rational dynamics with the scaling parameters above give pairs of partial differential equations $\mathbf{F}\left(\epsilon, u, u_{x}, \ldots\right)=0$ as the leading terms, and the error terms $\mathbf{F}^{1}\left(\epsilon, u, u_{x}, \ldots\right)=0$ by use of Taylor expansions (1.C).

Let us state our main theorem. The following comparison method discovers very rough structural similarity among different partial differential equations: 
Theorem 1.1. Let $f$ and $g$ be both relatively elementary and increasing functions of $n$ variables, which are mutually tropically equivalent. Let $\mathbf{F}$ and $\mathbf{G}$ be their leading terms of order at most $\alpha \geq 0$, and take positive $C^{\alpha+1}$ solutions $u, v:(0, \infty) \times\left[0, T_{0}\right) \rightarrow(0, \infty)$ with:

$$
\mathbf{F}\left(\epsilon, u, u_{x}, u_{s}, \ldots, u_{\alpha x}, u_{\alpha s}\right)=0, \mathbf{G}\left(\epsilon, v, v_{x}, v_{s}, \ldots, v_{\alpha x}, v_{\alpha s}\right)=0 .
$$

Assume both $u$ and $v$ are $\epsilon_{0}$ controlled bounded by $C$. Then for any $0<\epsilon \leq \min \left(\frac{1}{2 C}, \epsilon_{0}\right)$ and $D=\max (p, q)$, the estimates hold:

$$
\left(\frac{u(x, s)}{v(x, s)}\right)^{ \pm 1} \leq(2 M)^{8 \frac{c^{\epsilon^{-D}(x+k s)+1}-1}{c-1}}\left([u: v]_{(L+1) \epsilon}\right)^{c^{\epsilon^{-D}(x+k s)+n}} .
$$

From this we will induce various estimates in concrete examples with respect to their higher derivative rates below. We notice that as a general principle, double exponential growth are optimal in our setting (remark (2) in 2.C).

Now what are the rest is to find suitable pairs of PDEs which arise from the same automata, or in other words, to find suitable rational functions which produce the desired PDEs. This is the key step for our general machinery of discritization of PDE.

Let us see explicit estimates for concrete cases. Here we treat two equations, one is quasi linear equations of order 1, and the other is diffusion equations of order 2 . The proofs contain two fundamental techniques, where one is cancellation, and the other is linear deformation both for rational functions. They are obtained by combinations of results in section 4 with lemma 3.3 .

Firstly let us consider the quasi linear equations, and choose the uniform norm of second order:

$$
\|u\|_{2}=\max \left\{\left\|\frac{\partial^{2} u}{\partial x^{2}}\right\|_{C^{0}},\left\|\frac{\partial^{2} u}{\partial s^{2}}\right\|_{C^{0}},\left\|\frac{\partial^{2} u}{\partial x \partial s}\right\|_{C^{0}}\right\}
$$

We put the second derivative rates $K(u)=\frac{\|u\|_{2}}{\inf _{(x, s) \in(0, \infty) \times\left[0, T_{0}\right)} u(x, s)}$.

Let us fix any positive constant $K_{0}>0$.

Theorem 1.2. For any $0<\epsilon \leq 0.1 K_{0}^{-1}$, let $v, u:(0, \infty) \times\left[0, T_{0}\right) \rightarrow(0, \infty)$ be $C^{2}$ solutions to the quasi linear equations:

$$
v_{s}+\epsilon v v_{x}-\frac{1}{2} v^{2}=0, \quad 2 u_{s}+\epsilon u\left(u_{s}+u_{x}\right)=0 .
$$


Suppose their second derivative rates are bounded by $K_{0} \geq K(u), K(v)$. Then they satisfy the asymptotic estimates for all $(x, s) \in(0, \infty) \times\left[0, T_{0}\right)$ :

$$
\left(\frac{u(x, s)}{v(x, s)}\right)^{ \pm 1} \leq 40^{2^{-1}(x+2 s)+4}\left([u: v]_{2 \epsilon}\right)^{2^{\epsilon^{-1}(x+2 s)+3}} .
$$

In particular when $u(x, s) \equiv R>0$ is constant, then the estimates hold:

$$
\begin{aligned}
R(40)^{-2^{\epsilon^{-1}(x+2 s)+4}}\left([v: R]_{2 \epsilon}\right)^{-2^{\epsilon^{-1}(x+2 s)+3}} & \\
& \leq v(x, s) \leq R(40)^{2^{\epsilon^{-1}(x+2 s)+4}}\left([v: R]_{2 \epsilon}\right)^{2^{\epsilon^{-1}(x+2 s)+3}} .
\end{aligned}
$$

Next we treat diffusion equations. Let $F$ be an elementary and increasing function. Here we consider the diffusion equations of the type:

$$
u_{s}=u_{2 x}+F(u) \text {. }
$$

There has been various studies for such type of diffusion equations, in relation with blowing up of solutions. We point out two known results.

(1) Let $F(u)=u^{l}$ for $l=1,2, \ldots$ If $l=2$, then any positive solutions to the equation blow up at finite time. For $l \geq 4$, it has global positive solutions for small initial values $([\mathrm{F}])$. The number 3 is called the Fujita index (for one dimensional case).

(2) For all $l$, if the initial functions take sufficiently large values, then such solutions blow up at fintie time ([LN]).

For this case we take the uniform norm of the third derivatives:

$$
\|u\|_{3}=\max \left\{\left\|\frac{\partial^{3} u}{\partial x^{3}}\right\|_{C^{0}},\left\|\frac{\partial^{3} u}{\partial s^{3}}\right\|_{C^{0}},\left\|\frac{\partial^{3} u}{\partial x^{2} \partial s}\right\|_{C^{0}},\left\|\frac{\partial^{3} u}{\partial x \partial s^{2}}\right\|_{C^{0}}\right\} .
$$

Then we put the third derivative rates $K(u) \equiv \frac{\|u\|_{3}}{\inf _{(x, s) \in(0, \infty) \times\left[0, T_{0}\right)} u(x, s)}$.

Firstly let us compare linear diffusion equations with advection-diffusion equations of variable exchange. For the linear case, the corresponding Lipschitz constant is equal to one, and one obtains the exponential asymptotics:

Proposition 1.3. Let us fix $K_{0}>0$, and choose any $0<\epsilon \leq\left(200 K_{0}\right)^{-1}$. Let $u, v:(0, \infty) \times[0, \infty) \rightarrow(0, \infty)$ be $C^{3}$ solutions to the linear equations:

$$
\frac{7}{5} u_{s}-\frac{193}{40} u_{2 x}=0, \quad \frac{15}{8} \epsilon v_{s}+\frac{43}{32} v_{x}-\frac{19}{16} \epsilon^{3} v_{2 s}=0 .
$$


Suppose the third derivative rates satisfy the bounds $K(u), K(v) \leq K_{0}$. Then they satisfy the exponential asymptotic estimates for all $(x, s) \in(0, \infty) \times$ $[0, \infty)$ :

$$
\left(\frac{u(x, s)}{v(x, s)}\right)^{ \pm 1} \leq 10^{48\left(\epsilon^{-2}(x+4 s)+1\right)}[u: v]_{5 \epsilon} .
$$

This is obtained by applying tropical linear deformation of rational functions. Such method is also applied for non linear case as below.

For $1<a \in \mathbb{Q}$, let us consider the diffusion equations of the form:

$$
u_{s}=u_{2 x}+u^{a} .
$$

Let us consider the special solution $v:\left[0, S_{0}\right) \rightarrow(0, \infty)$ given by:

$$
v(s)=\frac{c}{\left(1-c^{a-1}(a-1) s\right)^{(a-1)^{-1}}}
$$

where $S_{0}=\frac{1}{c^{a-1}(a-1)}$. Both $v$ and its third derivative are increasing functions. Thus for any $0<s_{0}<S_{0}$ and $\alpha=(a-1)^{-1}$, the third derivative rate $K\left(s_{0}\right)$ for the restriction $v:\left[0, s_{0}\right] \rightarrow(0, \infty)$ is bounded by:

$$
K\left(s_{0}\right)=\frac{c^{3 \alpha^{-1}}(\alpha+1)(\alpha+2)}{\alpha^{2}\left(1-c^{\alpha^{-1}} \alpha^{-1} s_{0}\right)^{\alpha+3}}
$$

(Remark in 4.B.3). Conversely for any $K(0) \leq K_{0}<\infty$, there are unique $s_{0}<S_{0}$ so that the equalities $K_{0}=K\left(s_{0}\right)$ hold.

Theorem 1.4. Let us fix any $K_{0}=K\left(s_{0}\right)$. For any $1<a \in \mathbb{Q}$ and $T_{0} \leq s_{0}$, let $u:(0, \infty) \times\left[0, T_{0}\right) \rightarrow(0, \infty)$ be $C^{3}$ solutions to the diffusion equations:

$$
u_{s}-u_{2 x}=u^{a} .
$$

Suppose their third derivative rates are bounded by $K_{0}$. Then for any $0<\epsilon \leq\left(200 K_{0}\right)^{-1}$, u satisfy the asymptotic estimates:

$$
\left(\frac{u(x, s)}{v(s)}\right)^{ \pm 1} \leq 10^{40 \frac{a^{\epsilon^{-2}(2 x+4 s)+1}-1}{a-1}}\left([u: v]_{5 \epsilon}\right)^{a^{\epsilon^{-2}(2 x+4 s)+4}} .
$$

Next we treat diffusion equations of the form:

$$
u_{s}-u_{2 x}-u^{a}-\delta u^{b}=0, \quad(1<a<b, 0<\delta<<1)
$$


where we consider the equations of the types:

$$
(a, b)=(2,3),\left(1+\alpha^{-1}, 1+2 \alpha^{-1}\right)
$$

and $0.5<\alpha<1$ are any rational numbers. Both the right and left hand side terms touch the Fujita index $(=3)$, and the middle terms cross it. For example it contains the case $(a, b)=(2.5,4)$.

For $0.5 \leq \alpha \leq 1$ and $c>0$, let us put:

$$
\begin{aligned}
& K\left(s_{0}\right)=\frac{c^{3 \alpha^{-1}} 5^{3}(\alpha+1)(\alpha+2)}{6^{3} \alpha^{2}\left(1-c^{\prime} s_{0}\right)^{\alpha+3}}\left(0 \leq s_{0}<\frac{1}{c^{\prime}}\right), \quad c^{\prime}=\frac{5 c^{\alpha^{-1}}}{6 \alpha}, \\
& (a, b)=\left(1+\alpha^{-1}, 1+2 \alpha^{-1}\right), \quad \delta=\mu \epsilon^{2}, \quad \mu=\frac{\alpha+1}{9 \alpha} .
\end{aligned}
$$

For any positive rational numbers $\mu=\frac{p}{q} \in \mathbb{Q}_{>0}$, where $p, q \in \mathbb{N}$ are relatively prime, we put $c_{\mu} \equiv p q \in \mathbb{Z}_{>0}$.

Let us compare $u$ with the function:

$$
v(s)=\frac{c}{\left(1-c^{\prime} s\right)^{\alpha}} .
$$

Let us fix any $K_{0}=K\left(s_{0}\right) \geq K(0)$.

Theorem 1.5. For any $0<T_{0} \leq s_{0}$ and any $0<\epsilon \leq\left(200 K_{0}\right)^{-1}$, let $u:(0, \infty) \times\left[0, T_{0}\right) \rightarrow(0, \infty)$ be $C^{3}$ solutions to the the diffusion equations:

$$
u_{s}-u_{2 x}=u^{a}+\delta u^{b} .
$$

Suppose their third derivative rates are bounded by $K_{0}$. Then $u$ satisfy the asymptotic estimates:

$$
\left(\frac{u(x, s)}{v(s)}\right)^{ \pm 1} \leq\left(2 M_{\mu}\right)^{8^{\frac{\epsilon^{-2}(2 x+4 s)+1}{b-1}}}\left([u: v]_{5 \epsilon}\right)^{b^{\epsilon^{-2}(2 x+4 s)+4}}
$$

where $M_{\mu}=\max \left(2 \times 10^{3} c_{\mu}^{2}, 3 \times 10^{4}\right)$.

These results come from a general procedure of comparison method which we will describe below. Our task is to seek for discrete dynamics which induce desired PDE, but such dynamics are not unique. The analytic conditions in these results are heavily depend on choices of such discrete dynamics. Particularly of interest for us is to obtain such estimates by use of $\alpha+1$ 
derivatives for larger $\alpha$. If one can find more suitable discrete dynamics, then one will obtain better asymptotic estimates of solutions.

On the other hand in [KT] we have constructed some examples of pairs of PDEs whose particular solutions do not have such uniform bounds mutually, and so which do not arise from the above procedure. Thus our relations on the uniform bounds for solutions are non trivial among the set of PDEs of 2 variables.

1.A.2 Uniform bounds of higher derivative rates: Functions we consider here are assumed to satisfy uniform boundedness of higher derivatives rates. Functions 'close' to polynomials will be particular cases.

It follows from the next lemma that there are pairs of $C^{\alpha+1}$ functions which admit uniformly bounded derivative rates of order $\alpha+1$, and still break these double exponential estimates on any large bounded domains:

Lemma 1.6. Let us take any $\alpha \geq 1$ and any large $C_{0}>>0$. Then there are constants $c_{\alpha+1}$ independent of $C_{0}$, and pairs of $C^{\alpha+1}[0,2)$ functions $u, v$ whose derivative rates of order $\alpha+1$ are bounded by $c_{\alpha+1}$, so that the estimates hold:

$$
\frac{v(s)}{u(s)} \begin{cases}=1 & 0 \leq s \leq 1 \\ \geq C_{0} & 2-\left(2 C_{0}\right)^{-1} \leq s<2 .\end{cases}
$$

Proof: Let $u:[0,2) \rightarrow\left(0,2 C_{0}\right]$ be the linear function by $u(s)=C_{0}(2-x)$. $\alpha+1$ derivatives of $u$ vanish for $\alpha \geq 1$, and so all higher derivative rates are zero. We construct $v:[0,2) \rightarrow\left[0.5 C_{0}, 2 C_{0}\right]$ which satisfies:

$$
v(s)= \begin{cases}u(s) & 0 \leq s \leq 1 \\ 0.5 C_{0} & 1.5 \leq s \leq 2\end{cases}
$$

Let $w:[0,2) \rightarrow[0.5,2]$ be a smooth and non increasing function which satisfies $w(s)=2-s$ for $0 \leq s \leq 1$, and $\equiv 0.5$ for $1.5 \leq s \leq 2$. Then there are constants $c_{\alpha+1}$ so that its derivative rates of order $\alpha+1$ are bounded by $c_{\alpha+1}$. Let us put $v:[0,2) \rightarrow\left[0.5 C_{0}, 2 C_{0}\right]$ by $v(s)=C_{0} w(s)$. Then $v$ are the desired functions, since they have the same higher derivative rates as $w$. This completes the proof.

When the domains for $(x, s)$ are unbounded, what we are focusing by those double exponential estimates, is not behavior at infinity for $(x, s)$. In fact it follows from the assumption of uniform boundedness of higher 
derivative rates that $u$ will grow at most exponentially. So constrains of the defining equations have an effective influence on bouneded regions.

1.B Real rational dynamics and tropical geometry: A relative $(\max ,+)$-function $\varphi$ is a piecewise linear function of the form:

$$
\varphi(\bar{x})=\max \left(\alpha_{1}+\bar{a}_{1} \bar{x}, \ldots, \alpha_{m}+\bar{a}_{m} \bar{x}\right)-\max \left(\beta_{1}+\bar{b}_{1} \bar{x}, \ldots, \beta_{l}+\bar{b}_{l} \bar{x}\right)
$$

where $\bar{a}_{l} \bar{x}=\sum_{i=1}^{n} a_{l}^{i} x_{i}, \bar{x}=\left(x_{1}, \ldots, x_{n}\right) \in \mathbb{R}^{n}, \bar{a}_{l}=\left(a_{l}^{1}, \ldots, a_{l}^{n}\right), \bar{b} \in \mathbb{Z}^{n}$ and $\alpha_{i}, \beta_{i} \in \mathbb{R}$. We say that the multiple integer $M \equiv m l$ is the number of the components of $\varphi$.

Correspondingly tropical geometry associates the parametrized rational function given by (see [Mi]):

$$
f_{t}(\bar{z}) \equiv \frac{k_{t}(\bar{z})}{h_{t}(\bar{z})}=\frac{\sum_{k=1}^{m} t^{\alpha_{k}} \bar{z}^{\bar{a}_{k}}}{\sum_{k=1}^{l} t^{\beta_{k}} \bar{z}^{\bar{b}_{k}}}
$$

where $\bar{z}^{\bar{a}}=\prod_{i=1}^{n} z_{i}^{a^{i}}, \bar{z}=\left(z_{1}, \ldots, z_{n}\right) \in \mathbb{R}_{>0}^{n}$. We say that $f_{t}$ above is a relative elementary function. We say that both terms $h_{t}(\bar{z})=\Sigma_{k=1}^{l} t^{\beta_{k}} \bar{z}^{\bar{b}_{k}}$ and $k_{t}(\bar{z})=\sum_{k=1}^{m} t^{\alpha_{k}} \bar{z}^{\bar{a}_{k}}$ are just elementary functions.

These two functions $\varphi$ and $f_{t}$ admit one to one correspondence between their presentations. Moreover the defining equations are transformed by two steps, firstly taking conjugates by $\log _{t}$ and secondly by letting $t \rightarrow \infty$. Notice that when all $a_{i}$ and $b_{j}$ are zero, then the corresponding $f_{t}$ are $t$ independent.

In some cases the same $(\max ,+)$ function admits different presentations, while the corresponding rational functions are mutually different. For example for $\varphi(x) \equiv \max (x, x)=x \equiv \psi(x)$, the corresponding rational functions $f_{t}(z)=2 z$ and $g_{t}(z)=z$ are mutually different. We call such a pair of rational functions tropically equivalent.

Let $f_{t}: \mathbb{R}_{>0}^{n} \rightarrow(0, \infty)$ be a rarional function, and consider the discrete dynamics defined by:

$$
z_{N}=f_{t}\left(z_{N-n}, \ldots, z_{N-1}\right), \quad N \geq n
$$

with initial values $\left(z_{0}, \ldots, z_{n-1}\right) \in \mathbb{R}_{>0}^{n}$. One can regard that tropically equivalent rational functions determine the same dynamics at infinity.

Let us put:

$$
P_{N}(c)= \begin{cases}\frac{c^{N-n+1}-1}{c-1} & c>1 \\ N-n+1 & c=1\end{cases}
$$


For a relative elementary function $f_{t}$, let $c_{f} \geq 1$ be the Lipschitz constant and $M_{f}$ be the number of the components with respect to the corresponding (max, +)-function.

Our basic analysis on the orbits is given by the following (corollary 2.8):

Lemma 1.7. Let $g_{t}$ be tropically equivalent to $f_{t}$, and $\left\{z_{N}\right\}_{N}$ and $\left\{w_{N}\right\}_{N}$ be the orbits for $f_{t}$ and $g_{t}$ with the initial values $\bar{z}_{0}=\left(z_{0}, \ldots, z_{n-1}\right)$ and $\bar{w}_{0}=\left(w_{0}, \ldots, w_{n-1}\right)$ respectively. Then the estimates hold:

$$
\left(\frac{z_{N}}{w_{N}}\right)^{ \pm} \leq M^{4 P_{N}(c)}\left[\max _{0 \leq i \leq n-1}\left(\frac{z_{i}}{w_{i}}\right)^{ \pm 1}\right]^{c^{N}}
$$

where $c=\max \left(c_{f}, c_{g}\right)$ and $M=\max \left(M_{f}, M_{g}\right)$.

If the initial values are the same, then uniform estimates hold (prop 2.3):

$$
\left(\frac{z_{N}}{w_{N}}\right)^{ \pm} \leq M^{2 P_{N}(c)}
$$

One particular feature is that when the Lipschitz constant is equal to 1, then the above inequalites give the exponential estimates, while for $c>1$, they are double exponential. The former is applied for the estimates of solutions to linear PDEs.

When one considers evolutional discrete dynamics, a parallel estimates are given. An evolutional discrete dynamics is given by flows of the form $\left\{z_{N}^{t}\right\}_{t, N \geq 0}$, where $t$ is time parameter. A general equation of evolutional discrete dynamics is of the form:

$$
z_{N+1}^{t+1}=f\left(z_{N-l_{0}}^{t+1}, \ldots, z_{N}^{t+1}, z_{N-l_{1}}^{t}, \ldots, z_{N+k_{1}}^{t}, \ldots, z_{N+k_{d+1}}^{t-d}\right)
$$

where $l_{i}, k_{j} \geq 0, N \geq \max \left(l_{0}, \ldots, l_{d+1}\right)$ and $t \geq d$, with initial values:

$$
\bar{z}_{0}^{0} \equiv\left\{z_{a}^{t}\right\}_{0 \leq a \leq \max \left(l_{0}, \ldots, l_{d+1}\right), t=0,1, \ldots} \cup\left\{z_{N}^{h}\right\}_{0 \leq h \leq d, N=0,1, \ldots} .
$$

Let us take $g$ tropically equivalent to $f$, and consider the dynamics $\left\{w_{n}^{t}\right\}$ defined by $g$ with any initial values $\bar{w}_{0}^{0}$. Then we put the initial rates by:

$$
\left[\bar{z}_{0}^{0}: \bar{w}_{0}^{0}\right] \equiv \sup _{0 \leq a \leq \max \left(l_{0}, \ldots, l_{d+1}\right), b=0,1, \ldots, \text { or } a=0,1, \ldots, 0 \leq b \leq d}\left\{\frac{z_{a}^{b}}{w_{a}^{b}}, \frac{w_{a}^{b}}{z_{a}^{b}}\right\} .
$$

Let us put $l=\max \left(l_{0}, l_{1}, \ldots, l_{d+1}\right), k=\max \left(k_{1}, \ldots, k_{d+1}\right)$ and

$$
A(N, t) \equiv(t-d-1) k+N-l+n-1
$$

for $N \geq l+1$ and $t \geq d+1$. 
Proposition 1.8. (1) Let $f$ and $g$ be tropically equivalent. Then any orbits $\left\{z_{N}^{t}\right\}_{N}$ and $\left\{w_{N}^{t}\right\}_{N}$ for $f$ and $g$ with the initial values $\bar{z}_{0}^{0}$ and $\bar{w}_{0}^{0}$ respectively, satisfy the estimates:

$$
\left(\frac{z_{N}^{t}}{w_{N}^{t}}\right)^{ \pm} \leq M^{4 P_{A(N, t)}(c)}\left[\bar{z}_{0}^{0}: \bar{w}_{0}^{0}\right]^{c^{A(N, t)}}
$$

where $c=\max \left(c_{f}, c_{g}\right)$ and $M=\max \left(M_{f}, M_{g}\right)$.

(2) Let $f, f^{\prime}, g, g^{\prime}$ be four relative elementary functions, and assume that they are all monotone increasing and all tropically equivalent. Let $\left\{v_{N}^{t}\right\}_{N, t}$ and $\left\{u_{N}^{t}\right\}_{N, t}$ be positive sequences so that these satisfy the estimates:

$$
\begin{aligned}
& f^{\prime}\left(v_{N-l_{0}}^{t+1}, \ldots, v_{N+k_{d+1}}^{t-d}\right) \leq v_{N+1}^{t+1} \leq f\left(v_{N-l_{0}}^{t+1}, \ldots, v_{N+k_{d+1}}^{t-d}\right), \\
& g^{\prime}\left(u_{N-l_{0}}^{t+1}, \ldots, u_{N+k_{d+1}}^{t-d}\right) \leq u_{N+1}^{t+1} \leq g\left(u_{N-l_{0}}^{t+1}, \ldots, u_{N+k_{d+1}}^{t-d}\right)
\end{aligned}
$$

for all $N, t$. Then the ratios satisfy the uniform estimates:

$$
\left(\frac{v_{N}^{t}}{u_{N}^{t}}\right)^{ \pm 1} \leq M^{8 P_{A(N, t)}(c)}\left[\bar{u}_{0}^{0}: \bar{v}_{0}^{0}\right]^{c^{A(N, t)}} .
$$

Here also if the Lipschitz constants $c$ are equal to 1 , then the above two estimates are at most exponential, while for the case $c>1$, they are double exponential.

Such general form will allow us to treat wider classes of PDE. But for concrete cases, we use evolutional discrete dynamics only of the forms:

$$
z_{N+1}^{t+1}= \begin{cases}f\left(z_{N-1}^{t+1}, z_{N}^{t}, z_{N+2}^{t}\right) & \text { for quasi linear equations } \\ f\left(z_{N}^{t}, z_{N+4}^{t}, z_{N-4}^{t-1}, z_{N-1}^{t-4}\right) & \text { for diffusion equations. }\end{cases}
$$

For the former $l=1, k=2, d=0$, and for the latter $l=k=d=4$. So they are given by:

$$
A(N, t)= \begin{cases}2 t+N-1 & (N \geq 2, t \geq 1) \\ 4 t+N-21 & (N \geq 5, t \geq 5)\end{cases}
$$

1.C Rough approximations by discrete dynamics: Let us describe our general procedure for approximating solutions to differential equations by discrete dynamics, and outline how to verify theorems in 1.A. 
Let us consider a $C^{\alpha+1}$ function $u:(0, \infty) \rightarrow(0, \infty)$, and for $1 \leq|i| \leq$ $n-1$, take the Taylor expansions:

$$
u(x+i \epsilon)=u(x)+i \epsilon u_{x}+\frac{(i \epsilon)^{2}}{2} u_{2 x}+\cdots+\frac{(i \epsilon)^{\alpha}}{\alpha !} u_{\alpha x}+\frac{(i \epsilon)^{(\alpha+1)}}{(\alpha+1) !} u_{(\alpha+1) x}\left(\xi_{i}\right) .
$$

Then for small $\epsilon>0$ and $N=0,1,2, \ldots$, let us put

$$
z_{N} \equiv \epsilon u(\epsilon N)=\epsilon u(x), \quad\left(N=\frac{x}{\epsilon}\right) .
$$

Let $f=\frac{k}{h}: \mathbb{R}_{>0}^{n} \rightarrow(0, \infty)$ be a relative elementary function of $n$ variables, where both $h$ and $k$ are elementary, and consider the discrete dynamics defined by $w_{N+1}=f\left(w_{N-n+1}, \ldots, w_{N}\right)$ with the initial value $w_{i}=\epsilon u(\epsilon i)>0$ for $0 \leq i \leq n-1$. Our basic idea is to regard that the sequence $\left\{w_{N}\right\}_{N}$ would approximate the orbit $\left\{z_{N}\right\}_{N}$.

So let us consider the difference and insert the Taylor expansions:

$$
\begin{aligned}
& z_{N+1}-f\left(z_{N-n+1}, \ldots, z_{N}\right)=\epsilon u(x+\epsilon)-f(\epsilon u(x-(n-1) \epsilon), \ldots, \epsilon u(x)) \\
& =\epsilon\left(u+\epsilon u_{x}+\frac{\epsilon^{2}}{2} u_{2 x}+\ldots\right)-f\left(\epsilon\left(u-(n-1) \epsilon u_{x}+\ldots\right), \ldots, \epsilon u\right) \\
& =\frac{\epsilon F^{1}(u)+\epsilon^{2} F^{2}\left(u_{x}\right)+\epsilon^{3} F^{3}\left(u, u_{x}\right)+. .+\epsilon^{m} F^{m^{\prime}}\left(u, . ., u_{(\alpha+1) x}(\xi)\right)+. .}{h(\epsilon u(x-(n-1) \epsilon), \ldots, \epsilon u(x))}
\end{aligned}
$$

where $F^{k}$ are monomials.

For any finite subset $A \subset\{1,2,3, \ldots\}$, let us divide the expanded sum into two terms as:

$$
\begin{aligned}
& =\frac{\sum_{i \in A} \epsilon^{s_{i}} F^{s_{i}^{\prime}}\left(u, u_{x}, \ldots, u_{\alpha x}\right)}{h(\epsilon u(x-(n-1) \epsilon), \ldots)}+\frac{\Sigma_{j \in A^{c}} \epsilon^{s_{j}} F^{s_{j}^{\prime}}\left(u, u_{x}, \ldots, u_{(\alpha+1) x}(\xi)\right)}{h(\epsilon u(x-(n-1) \epsilon), \ldots)} \\
& \equiv \mathbf{F}\left(\epsilon, u, u_{x}, \ldots, u_{\alpha x}\right)+\epsilon^{2} \mathbf{F}^{1}\left(\epsilon, u, u_{x}, . ., u_{(\alpha+1) x}\left(\xi_{1}\right), . ., u_{(\alpha+1) x}\left(\xi_{n-1}\right)\right)
\end{aligned}
$$

We say that $\mathbf{F}$ and $\mathbf{F}^{1}$ are the leading and error terms respectively. Once one has chosen a relative elementary function $f$, then the above process determines a PDE defined by $\mathbf{F}$, while tropical geometry gives an automaton by a $(\max ,+)$ function $\varphi$. So $f$ plays a role of a bridge to connect between PDE and automaton. 
Let us define $\epsilon$ variation of $\mathbf{F}^{1}$ by:

$$
\begin{aligned}
& \left\|\mathbf{F}^{1}\left(\epsilon, u, u_{x}, \ldots, u_{\alpha x}, u_{(\alpha+1) x}\left(\xi_{1}\right), \ldots, u_{(\alpha+1) x}\left(\xi_{n-1}\right)\right)\right\|_{\epsilon}(x) \equiv \\
& \quad \sup _{\mu_{i}-x \in I(n, \epsilon)}\left|\mathbf{F}^{1}\left(\epsilon, u(x-\epsilon), . ., u_{\alpha x}(x-\epsilon), u_{(\alpha+1) x}\left(\mu_{1}\right), . ., u_{(\alpha+1) x}\left(\mu_{n-1}\right)\right)\right|
\end{aligned}
$$

where $I(n, \epsilon)=[-n \epsilon, 0]$ is the fluctuation interval.

Let us say that a $C^{\alpha+1}$ function $u:(0, \infty) \rightarrow(0, \infty)$ is $\epsilon$ controlled, if there is some constant $C>0$ so that $\epsilon$ variation of $\mathbf{F}^{1}$ satisfy the pointwise estimates for all $x \in(0, \infty)$ :

$$
C u(x) \geq\left\|\mathbf{F}^{1}\left(\epsilon, u, u_{x}, \ldots, u_{\alpha x}, u_{(\alpha+1) x}\left(\xi_{1}\right), \ldots, u_{(\alpha+1) x}\left(\xi_{n-1}\right)\right)\right\|_{\epsilon}(x) .
$$

For two functions $u, v$, we put their initial rates by $[u: v]_{\epsilon} \equiv \sup _{x \in(0, \epsilon]}\left(\frac{u(x)}{v(x)}\right)^{ \pm 1}$.

Now we state the first estimates. Let $f$ and $g$ be relatively elementary functions, and $\mathbf{F}$ and $\mathbf{G}$ be their corresponding leading terms. Recall that associated with $f$ are the Lipschiz constant $c_{f} \geq 1$ and the number of the components $M_{f}$. Let us put $c=\max \left(c_{f}, c_{g}\right)$ and $M=\max \left(M_{f}, M_{g}\right)$.

Proposition 1.9. Let $f$ and $g$ be both relatively elementary and increasing functions of $n$ variables, which are mutually tropically equivalent. Let $\mathbf{F}$ and $\mathbf{G}$ be their leading terms of order at most $\alpha \geq 0$, and take positive $C^{\alpha+1}$ solutions $u, v:(0, \infty) \rightarrow(0, \infty)$ with:

$$
\mathbf{F}\left(\epsilon, u, u_{x}, \ldots, u_{\alpha x}\right)=0, \quad \mathbf{G}\left(\epsilon, v, v_{x}, \ldots, v_{\alpha x}\right)=0 .
$$

Assume both $u$ and $v$ are $\epsilon_{0}$ controlled bounded by $C$. Then for any $0<\epsilon \leq \min \left(\frac{1}{2 C}, \epsilon_{0}\right)$, the estimates hold:

$$
\left(\frac{u(x)}{v(x)}\right)^{ \pm 1} \leq(2 M)^{8 \frac{c^{\epsilon^{-1} x+1}-1}{c-1}}\left([u: v]_{n \epsilon}\right)^{c^{\epsilon^{-1} x+1}} .
$$

One can proceed parallelly for evolutional case. Let $f$ be a relatively elementary function, and consider the evolutional discrete dynamics defined by the equation $z_{N+1}^{t+1}=f\left(z_{N-l_{0}}^{t+1}, \ldots, z_{N+k_{d+1}}^{t-d}\right)$.

Let us take a $C^{\alpha+1}$ function $u:(0, \infty) \times\left[0, T_{0}\right) \rightarrow(0, \infty)$, and introduce another parameters by:

$$
\epsilon^{m} u(x, s)=z_{N}^{t}, \quad N=\frac{x}{\epsilon^{p}}, \quad t=\frac{s}{\epsilon^{q}}
$$


where $p, q \geq 1$ and $m \geq 0$ are integers. By the same way as one variable case, one takes the Taylor expansion, and take the difference:

$$
\begin{aligned}
& z_{N+1}^{t+1}-f\left(z_{N-l_{0}}^{t+1}, \ldots, z_{N+k_{d+1}}^{t-d}\right) \\
& =\epsilon^{m} u\left(x+\epsilon^{p}, s+\epsilon^{q}\right)- \\
& \quad f\left(\epsilon^{m} u\left(x-l_{0} \epsilon^{p}, s+\epsilon^{q}\right), \ldots, \epsilon^{m} u\left(x+k_{d+1} \epsilon^{p}, s-d \epsilon^{q}\right)\right) \\
& =\frac{\epsilon^{m} F^{1}(u)+\epsilon^{m+p} F^{2}\left(u_{x}\right)+\epsilon^{m+q} F^{3}\left(u_{s}\right)+\epsilon^{2 m+p} F^{4}\left(u, u_{x}\right)+\ldots}{h\left(\epsilon^{m} u\left(x-l_{0} \epsilon^{p}, s+\epsilon^{q}\right), \ldots, \epsilon u\left(x+k_{d+1} \epsilon^{p}, s-d \epsilon^{q}\right)\right)} \\
& =\mathbf{F}\left(\epsilon, u, u_{x}, u_{s}, u_{x s}, \ldots, u_{\alpha x}, u_{\alpha s}\right) \\
& \quad+\epsilon^{m+1} \mathbf{F}^{1}\left(\epsilon, u(x, s), u_{x}(x, s), \ldots, u_{\alpha x}(x, s),\right. \\
& \left.\quad u_{s}(x, s), \ldots, u_{\alpha s}(x, s),\left\{u_{(\alpha+1) x}\left(\xi_{i j}\right), \ldots, u_{(\alpha+1) s}\left(\xi_{i j}\right)\right\}_{i, j}\right) .
\end{aligned}
$$

By the same way as before one defines the $\epsilon$ variation $\left\|\mathbf{F}^{1}\right\|_{\epsilon}(x, s)$ and $\epsilon_{0}$ controlledess (3.B). Combining this construction with proposition 1.9, one obtains theorem 1.1.

Our basic process goes as follows. Firstly we choose a $\operatorname{PDE} \mathbf{F}\left(u, u_{x}, u_{s}, \ldots\right)$ $=0$, and fix scaling parameters. Then find a relative elementary function $f$ which induces $\mathbf{F}$ as its leading term. Next take another relative elementary $g$ which is tropically equivalent to $f$. Then by use of the same scaling parameters, it induces its leading term $\mathbf{G}$. Finally for two solutions $u$ and $v$ with $\mathbf{F}\left(u, u_{x}, u_{s}, \ldots\right)=0$ and $\mathbf{G}\left(v, v_{x}, v_{s}, \ldots\right)=0$ respectively, we seek for analytic conditions to both $u$ and $v$ which insure $\epsilon_{0}$ controllednesss. Even though choice of $f$ and $g$ are rather flexible, whether one could find some reasonable conditions for solutions depends on choices of these functions.

\section{Discrete dynamics and tropical geometry}

2.A Elementary functions: A relative $(\max ,+)$-function $\varphi$ is a piecewise linear function of the form:

$$
\varphi(\bar{x})=\max \left(\alpha_{1}+\bar{a}_{1} \bar{x}, \ldots, \alpha_{m}+\bar{a}_{m} \bar{x}\right)-\max \left(\beta_{1}+\bar{b}_{1} \bar{x}, \ldots, \beta_{l}+\bar{b}_{l} \bar{x}\right)
$$

where $\bar{a}_{k} \bar{x}=\sum_{i=1}^{n} a_{k}^{i} x_{i}, \bar{x}=\left(x_{1}, \ldots, x_{n}\right) \in \mathbb{R}^{n}, \bar{a}_{k}=\left(a_{k}^{1}, \ldots, a_{k}^{n}\right), \bar{b}_{k} \in \mathbb{Z}^{n}$ and $\alpha_{k}, \beta_{k} \in \mathbb{R}$.

For each relative $(\max ,+)$ function $\varphi$ as above, we associate a parametrized rational function by:

$$
f_{t}(\bar{z})=\frac{\sum_{k=1}^{m} t^{\alpha_{k}} \bar{z}^{\bar{a}_{k}}}{\sum_{k=1}^{l} t^{\beta_{k}} \bar{z}^{\bar{b}_{k}}}
$$


where $\bar{z}^{\bar{a}_{k}}=\prod_{i=1}^{n} z_{i}^{a_{k}^{i}}, \bar{z}=\left(z_{1}, \ldots, z_{n}\right) \in \mathbb{R}_{>0}^{n}=\left\{\left(w_{1}, \ldots, w_{n}\right): w_{i}>0\right\}$.

We say that $f_{t}$ above is a relative elementary function. Notice that any relative elementary functions take positive values for $\bar{z} \in \mathbb{R}_{>0}^{n}$.

We say that the integer:

$$
M \equiv m l
$$

is the number of the components.

We say that $f_{t}(\bar{z})=\sum_{k=1}^{m} t^{\alpha_{k}} \bar{z}^{\bar{j}_{k}}$ is an elementary rational function $([\mathrm{K} 2])$. The corresponding $(\max ,+)$-function is given by $\varphi(\bar{x})=\max \left(\alpha_{1}+\overline{j_{1}} \bar{x}, \ldots, \alpha_{m}+\right.$ $\left.\bar{j}_{m} \bar{x}\right)$, and in this case $m$ is the number of the components.

These two functions $\varphi$ and $f_{t}$ are connected passing through some intermediate functions $\varphi_{t}([\mathrm{LiM}],[\mathrm{Mi}])$. Let us describe it shortly below. For $t>1$, there is a family of semirings $R_{t}$ which are all the real number $\mathbb{R}$ as sets. The multiplications and the additions are respectively given by $x \oplus_{t} y=\log _{t}\left(t^{x}+t^{y}\right)$ and $x \otimes_{t} y=x+y$. As $t \rightarrow \infty$ one obtains the equality:

$$
x \oplus_{\infty} y=\max (x, y) .
$$

By use of $R_{t}$ as coefficients, one has relative $R_{t}$-polynomials:

$$
\varphi_{t}(\bar{x})=\left(\alpha_{1}+\bar{a}_{1} \bar{x}\right) \oplus_{t} \cdots \oplus_{t}\left(\alpha_{m}+\bar{a}_{m} \bar{x}\right)-\left(\beta_{1}+\bar{b}_{1} \bar{x}\right) \oplus_{t} \cdots \oplus_{t}\left(\beta_{l}+\bar{b}_{l} \bar{x}\right)
$$

The limit is given by the relative $(\max ,+)$ function above:

$$
\lim _{t \rightarrow \infty} \varphi_{t}(\bar{x})=\varphi(\bar{x}) .
$$

Let us put $\log _{t}: \mathbb{R}_{>0}^{n} \rightarrow \mathbb{R}^{n}$ by $\left(z_{1}, \ldots, z_{n}\right) \rightarrow\left(\log _{t} z_{1}, \ldots, \log _{t} z_{n}\right)$. Then $\varphi_{t}$ and $f_{t}$ satisfy the following relation:

Proposition $2.1(\mathrm{LiM}, \mathrm{V}) \cdot f_{t} \equiv\left(\log _{t}\right)^{-1} \circ \varphi_{t} \circ \log _{t}: \mathbb{R}_{>0}^{n} \rightarrow(0, \infty)$ is the relative elementary function $f_{t}(\bar{z})=\sum_{k=1}^{m} t^{\alpha_{k}} \bar{z}^{\bar{a}_{k}} / \sum_{k=1}^{l} t^{\beta_{k}} \bar{z}^{\bar{b}_{k}}$.

These functions $\varphi, \varphi_{t}$ and $f_{t}$ admit one to one correspondence between their presentations. We say that $\varphi$ is the corresponding $(\max ,+)$-function to $f_{t}$. Notice that any relative $(\max ,+)$ functions of the form $\varphi(\bar{x})=$ $\max \left(\bar{a}_{1} \bar{x}, \ldots, \bar{a}_{m} \bar{x}\right)-\max \left(\bar{b}_{1} \bar{x}, \ldots, \bar{b}_{l} \bar{x}\right)$ correspond to $t$-independent relative elementary functions $f$.

2.B Discrete dynamics: Let $f_{t}: \mathbb{R}_{>0}^{n} \rightarrow(0, \infty)$ be a relative elementary function, and $\varphi$ be the corresponding ( $\max ,+)$-function. Let us consider the discrete dynamics defined by:

$$
z_{N}=f_{t}\left(z_{N-n}, \ldots, z_{N-1}\right), \quad N \geq n
$$


with initial values $\left(z_{0}, \ldots, z_{n-1}\right) \in \mathbb{R}_{>0}^{n}$. These orbits $\left\{z_{N}\right\}_{N}$ admit some asymptotic controll passing through tropical geometry, which we describe below. Let us compare the orbits $\left\{x_{N}\right\}_{N}$ with $\left\{z_{N}\right\}_{N}$, which are determined by:

$$
x_{N}=\varphi\left(x_{N-n}, \ldots, x_{N-1}\right)
$$

with the initial values $x_{0}=\log _{t} z_{0}, \ldots, x_{n-1}=\log _{t} z_{n-1}$. For this, we introduce the intermediate dynamics:

$$
x_{N}^{\prime}=\varphi_{t}\left(x_{N-n}^{\prime}, \ldots, x_{N-1}^{\prime}\right)
$$

with the same initial data $x_{0}^{\prime}=\log _{t} z_{0}, \ldots, x_{n-1}^{\prime}=\log _{t} z_{n-1}$.

By proposition 2.1, two orbits $\left\{z_{N}\right\}_{N}$ and $\left\{x_{N}^{\prime}\right\}_{N}$ are conjugate each other as $x_{N}^{\prime}=\log _{t} z_{N}$ for all $N=0,1, \ldots$ Since $\lim _{t \rightarrow \infty} \varphi_{t}=\varphi$ holds, one may think $\left\{\log _{t} z_{N}\right\}_{N}$ 'approximate' $\left\{x_{N}\right\}_{N}$ in some sense.

Let $\varphi$ and $\psi$ be two relative ( $\max ,+$ )-functions with $n$ variables. Then $\psi$ is equivalent to $\varphi$, if they are the same as maps, $\varphi\left(x_{1}, \ldots, x_{n}\right)=\psi\left(x_{1}, \ldots, x_{n}\right)$ for all $\left(x_{1}, \ldots, x_{n}\right) \in \mathbb{R}^{n}$ (but possibly they can have different presentations).

Definition 2.1 (K2). Let $f_{t}$ and $g_{t}$ be two relative elementary functions. $g_{t}$ is tropically equivalent to $f_{t}$, if the corresponding relative (max, + )-functions $\varphi$ and $\psi$ are equivalent.

Remarks: (1) If the pointwise estimate $\varphi \geq \varphi^{\prime}$ holds, then $\psi=\max \left(\varphi, \varphi^{\prime}\right)$ and $\varphi$ are equivalent. Let $f_{t}$ and $g_{t}$ be the corresponding relative elementary functions to $\varphi$ and $\varphi^{\prime}$ respectively. Then $h_{t} \equiv f_{t}+g_{t}$ is tropically equivalent to $f_{t}$.

(2) For any relative elementary $f_{t}$ and positive rational numbers $0<\alpha=$ $\frac{n}{m} \in \mathbb{Q}, \alpha f_{t}$ is tropically equivalent to $f_{t}$. In fact let $\varphi$ correspond to $f_{t}$. Then $n f_{t}$ are tropically equivalent to $f_{t}$, since $n f_{t}$ correspond to $\max (\varphi, \ldots, \varphi)=\varphi$ ( $n$ times). Similarly $\frac{1}{m} f_{t}$ are also tropically equivalent to $f_{t}$.

(3) For any tropically equivalent pairs of relative elementary functions $f_{t}$ and $g_{t}$, the corresponding $(\max ,+)$-functions $\varphi$ and $\psi$ have the same Lipschitz constant $c>0$, since they are the same as maps. On the other hand they may have different numbers of the components $M$ and $M^{\prime}$ since it depends on their presentations. For example if $f_{t}$ has $M$ number of the components, then $\frac{n}{m} f_{t}$ has $n m M$ number of the components.

(4) For our purposes in this paper, it is enough to treat the case that the Lipschitz constsnts $c$ for $\varphi$ is larger or equal to 1 , and later on we will assume the bounds $c \geq 1$. 
2.C Basic estimates and Lipschitz constants: Let $f_{t}: \mathbb{R}_{>0}^{n} \rightarrow(0, \infty)$ be a relative elementary function. Take initial values $\left(z_{0}, \ldots, z_{n-1}\right) \in \mathbb{R}_{>0}^{n}$, and consider the orbits $\left\{z_{N}\right\}_{N=0}^{\infty}$ defined by $z_{N}=f_{t}\left(z_{N-n}, \ldots, z_{N-1}\right)$ for $N \geq n$. Let $g_{t}$ be another relative elementary function, and consider its orbit $\left\{w_{N}\right\}_{N}$ with the same initial values $w_{i}=z_{i}$ for $0 \leq i \leq n-1$.

In order to estimate their asymptotic rates $\left(\frac{z_{N}}{w_{N}}\right)^{ \pm 1}$ in detail, we use the metric on $\mathbb{R}^{n}$ given by:

$$
d\left(\left(x_{0}, \ldots, x_{n-1}\right),\left(y_{0}, \ldots, y_{n-1}\right)\right) \equiv \max _{0 \leq i \leq n-1}\left\{\left|x_{i}-y_{i}\right|\right\}
$$

(which is of course equivalent to the standard one.)

Lemma 2.2. Let $f_{t}=f$ be t-independent, relative elementary and linear. Then the corresponding $(\max ,+)$-function $\varphi$ has its Lipschitz constant bounded by 1 .

Proof: This follows immediately, if one checks the estimates carefully. One can express $\varphi\left(x_{0}, \ldots, x_{n-1}\right)=\max \left(\alpha_{1}+x_{i_{1}}, \ldots, \alpha_{n-1}+x_{i_{n-1}}\right)-\max (0, \ldots, 0)$. Let $\varphi\left(x_{0}, \ldots, x_{n-1}\right)=\alpha_{1}+x_{i_{1}} \geq \varphi\left(y_{0}, \ldots, y_{n-1}\right)=\alpha_{2}+y_{i_{2}}$. Then the estimates hold:

$$
\begin{aligned}
& \left|\varphi\left(x_{0}, \ldots, x_{n-1}\right)-\varphi\left(y_{0}, \ldots, y_{n-1}\right)\right|=\alpha_{1}+x_{i_{1}}-\left(\alpha_{2}+y_{i_{2}}\right) \\
& \leq \alpha_{1}+x_{i_{1}}-\left(\alpha_{1}+y_{i_{1}}\right)=x_{i_{1}}-y_{i_{1}} \leq \max _{0 \leq i \leq n-1}\left\{\left|x_{i}-y_{i}\right|\right\} .
\end{aligned}
$$

This completes the proof.

In general we have double exponential estimates for $\left(\frac{z_{N}}{w_{N}}\right)^{ \pm 1}$ as below, but in a special case that the Lipschitz constants of the corresopnding $(\max ,+)$ functions are equal to 1, they can be improved to be just exponential. This happens when one considers linear PDE.

Let us put

$$
P_{N}(c)=\left\{\begin{array}{ll}
\frac{c^{N-n+1}-1}{c-1} & c>1, \\
(N-n+1) & c=1 .
\end{array} .\right.
$$

For a relative elementary function $f_{t}$, let $c_{f}$ be the Lipschitz constant and $M_{f}$ be the number of the components with respect to the corresponding $(\max ,+)$-function. 
Proposition 2.3. $f_{t}$ and $g_{t}$ are tropically equivalent, if and only if any orbits with the same intial values satisfy uniformly bounded rates:

$$
\left(\frac{z_{N}}{w_{N}}\right)^{ \pm 1} \equiv \frac{z_{N}}{w_{N}}, \frac{w_{N}}{z_{N}} \leq M^{2 P_{N}(c)}, \quad(N \geq n)
$$

where $c=\max \left(c_{f}, c_{g}\right)$ and $M=\max \left(M_{f}, M_{g}\right)$.

For the proof, we use the next lemma.

Let $\varphi(\bar{x})=\max \left(\alpha_{1}+\bar{a}_{1} \bar{x}, \ldots, \alpha_{m}+\bar{a}_{m} \bar{x}\right)-\max \left(\beta_{1}+\bar{b}_{1} \bar{x}, \ldots, \beta_{l}+\bar{b}_{l} \bar{x}\right)$ and $\varphi_{t}$ be the corresponding functions to $f_{t}$. For the same initial values $x_{0}=x_{0}^{\prime}, \ldots, x_{n-1}=x_{n-1}^{\prime}$, let us denote the orbits by $\left\{x_{N}\right\}_{N}$ and $\left\{x_{N}^{\prime}\right\}_{N}$ for $\varphi$ and $\varphi_{t}$ respectively.

We will improve lemma 2.2 in [K2] slightly.

Lemma 2.4. Let $c \geq 1$ and $M$ be the Lipschitz constant and the number of the components for $\varphi$ respectively. Then the estimates hold:

$$
\left|x_{N}-x_{N}^{\prime}\right| \leq P_{N}(c) \log _{t} M
$$

Proof: One can obtain the following estimates easily ([K2] lemma 2.1(1)):

$$
\left|\varphi\left(x_{0}, \ldots, x_{n-1}\right)-\varphi_{t}\left(x_{0}, \ldots, x_{n-1}\right)\right| \leq \log _{t} M \text {. }
$$

Let us denote $\bar{x}_{N}=\left(x_{N}, \ldots, x_{N+n-1}\right) \in \mathbb{R}^{n}$. Thus $x_{N+n}=\varphi\left(\bar{x}_{N}\right)$ hold for all $N \geq 0$. Similar for $\bar{x}_{N}^{\prime}$.

Firstly one has the estimates $\left|x_{n}^{\prime}-x_{n}\right| \leq \log _{t} M$ as above.

Since $\varphi$ is $c$-Lipschitz and $\bar{x}_{1}-\bar{x}_{1}^{\prime}=\left(0, \ldots, 0, x_{n}-x_{n}^{\prime}\right)$, the estimates:

$$
\begin{aligned}
\left|x_{n+1}-x_{n+1}^{\prime}\right| & =\left|\varphi\left(\bar{x}_{1}\right)-\varphi_{t}\left(\bar{x}_{1}^{\prime}\right)\right| \\
& \leq\left|\varphi\left(\bar{x}_{1}\right)-\varphi\left(\bar{x}_{1}^{\prime}\right)\right|+\left|\varphi_{t}\left(\bar{x}_{1}^{\prime}\right)-\varphi\left(\bar{x}_{1}^{\prime}\right)\right| \\
& \leq c\left|\bar{x}_{1}-\bar{x}_{1}^{\prime}\right|+\log _{t} M \leq(c+1) \log _{t} M
\end{aligned}
$$

hold. Next we have estimates:

$$
\begin{aligned}
& \left|\varphi\left(\bar{x}_{2}\right)-\varphi\left(\bar{x}_{2}^{\prime}\right)\right| \leq c \max \left(\left|x_{n+1}-x_{n+1}^{\prime}\right|,\left|x_{n}-x_{n}^{\prime}\right|\right) \leq c(c+1) \log _{t} M \\
& \left|x_{n+2}-x_{n+2}^{\prime}\right|=\left|\varphi\left(\bar{x}_{2}\right)-\varphi_{t}\left(\bar{x}_{2}^{\prime}\right)\right| \\
& \leq\left|\varphi\left(\bar{x}_{2}\right)-\varphi\left(\bar{x}_{2}^{\prime}\right)\right|+\left|\varphi\left(\bar{x}_{2}^{\prime}\right)-\varphi_{t}\left(\bar{x}_{2}^{\prime}\right)\right| \leq[c(c+1)+1] \log _{t} M
\end{aligned}
$$


The rest is just the repetition of the same process. Now suppose $c>1$. Then by a direct calculation, one obtains the estimates:

$$
\left|x_{N}-x_{N}^{\prime}\right| \leq \frac{c^{N-n+1}-1}{c-1} \log _{t} M .
$$

On the other hand when $c=1$, then $\left|x_{N}-x_{N}^{\prime}\right| \leq(N-n+1) \log _{t} M$ hold. This completes the proof.

Proof of proposition 2.3: The proof is almost the same as theorem 2.1 in [K2], but for convenience we will include only if part.

Let $\varphi$ and $\psi$ be the relative (max, + )-functions corresponding to $f_{t}$ and $g_{t}$ respectively. For the same initial values $x_{i}=y_{i}=\log _{t} z_{i}, 0 \leq i \leq n-1$, let us denote the corresponding orbits by $\left\{x_{N}\right\}_{N}$ and $\left\{y_{N}\right\}_{N}$. We also put $x_{N}^{\prime}=\log _{t}\left(z_{N}\right)$ and $y_{N}^{\prime}=\log _{t}\left(w_{N}\right)$ respectively. Thus $\left\{x_{N}^{\prime}\right\}_{N}$ is the orbit for $\varphi_{t}$ and $\left\{y_{N}^{\prime}\right\}_{N}$ is for $\psi_{t}$.

By lemma 2.4, the estimates:

$$
\left|x_{N}-x_{N}^{\prime}\right|,\left|y_{N}-y_{N}^{\prime}\right| \leq P_{N}(c) \log _{t} M
$$

hold. Suppose $f_{t}$ and $g_{t}$ are tropically equivalent, and so $\varphi$ and $\psi$ are the same as maps. Thus $x_{N}=y_{N}$ hold, and so we have the estimates:

$$
\log _{t}\left(\frac{z_{N}}{w_{N}}\right)^{ \pm} \leq\left|\log _{t}\left(z_{N}\right)-\log _{t}\left(w_{N}\right)\right|=\left|x_{N}^{\prime}-y_{N}^{\prime}\right| \leq 2 P_{N}(c) \log _{t} M .
$$

Thus we have the estimates:

$$
\left(\frac{z_{N}}{w_{N}}\right)^{ \pm} \leq \max \left(\frac{z_{N}}{w_{N}}, \frac{w_{N}}{z_{N}}\right) \leq M^{2 P_{N}(c)} .
$$

This completes the proof.

Remarks: (1) In order to determine $z_{N}$ for $N \geq n$, one needs to iterate $N-n+1$ times to apply function $f_{t}$. One can say that ratios between $N-n+1$ times iterations of $f_{t}$ and $g_{t}$ are at most uniformly double exponential rates.

(2) Such double exponential estimates are optimal between tropically equivalent functions. Let us consider two dynamics for $l, k \geq 1$ :

$$
z_{N}=f\left(z_{N-1}\right)=z_{N-1}^{l}, \quad w_{N}=g\left(w_{N-1}\right)=2 w_{N-1}^{k} .
$$

If $l=k$ holds, then $f$ and $g$ are tropically equivalent. Let $z_{0}=w_{0}$ be initial values. Then a direct calculation gives:

$$
z_{N}=z_{0}^{l^{N}}, \quad w_{N}=2^{\frac{k^{N}-1}{k-1}} w_{0}^{k^{N}}=2^{\frac{k^{N}-1}{k-1}} z_{0}^{k^{N}} .
$$


Thus if $l=k$, then the equality:

$$
\left(\frac{w_{N}}{z_{N}}\right)^{ \pm 1}=2^{ \pm \frac{l^{N}-1}{l-1}}
$$

holds, which satisfies the uniformly double exponential bound.

On the other hand if $k>l$, then

$$
\frac{w_{N}}{z_{N}}=2^{\frac{k^{N}-1}{k-1}} z_{0}^{k^{N}-l^{N}}
$$

which heavily depends on the initial values.

Lemma 2.5. Let $f_{t}$ and $g_{t}$ be relative elementary and assume that both are monotone increasing. Let $\left\{v_{N}\right\}_{N}$ be a positive sequence so that the estimates:

$$
g_{t}\left(v_{N-n}, \ldots, v_{N-1}\right) \leq v_{N} \leq f_{t}\left(v_{N-n}, \ldots, v_{N-1}\right), \quad N \geq n
$$

hold. Let $\left\{z_{N}\right\}_{N}$ and $\left\{w_{N}\right\}_{N}$ be two dynamics defined by $z_{N}=f_{t}\left(z_{N-n}, \ldots, z_{N-1}\right)$ and $w_{N}=g_{t}\left(w_{N-n}, \ldots, w_{N-1}\right)$ with the same initial value $z_{i}=w_{i}=v_{i}$ for $0 \leq i \leq n-1$ respectively. Then the estimates hold:

$$
w_{N} \leq v_{N} \leq z_{N} \quad(N=0,1, \ldots)
$$

Proof: We proceed by induction. For $N=n$, the estimates follows by the hypothesis. Suppose the estimates $w_{N} \leq v_{N} \leq z_{N}$ hold for $N \leq N_{0}-1$. Then the conclusion for $N_{0}$ follows from two estimates:

$$
\begin{aligned}
& w_{N_{0}}=g_{t}\left(w_{N_{0}-n}, \ldots, w_{N_{0}-1}\right) \leq g_{t}\left(v_{N_{0}-n}, \ldots, v_{N_{0}-1}\right), \\
& z_{N_{0}}=f_{t}\left(z_{N_{0}-n}, \ldots, z_{N_{0}-1}\right) \geq f_{t}\left(v_{N_{0}-n}, \ldots, v_{N_{0}-1}\right)
\end{aligned}
$$

and the assumption $g_{t}\left(v_{N_{0}-n}, \ldots, v_{N_{0}-1}\right) \leq v_{N_{0}} \leq f_{t}\left(v_{N_{0}-n}, \ldots, v_{N_{0}-1}\right)$.

This completes the proof.

Corollary 2.6. Let $f_{t}$ and $g_{t}$ be tropically equivalent, and assume the conditions in lemma 2.4 are satisfied. Then the estimates hold:

$$
\left(\frac{z_{N}}{v_{N}}\right)^{ \pm 1}, \quad\left(\frac{w_{N}}{v_{N}}\right)^{ \pm 1} \leq M^{2 P_{N}(c)}
$$

where $c=\max \left(c_{f}, c_{g}\right)$ and $M=\max \left(M_{f}, M_{g}\right)$. 
Proof: By lemma 2.5, the estimates $w_{N} \leq v_{N} \leq z_{N}$ hold for all $N=$ $0,1, \ldots$ On the other hand by proposition 2.3 , the uniform bounds $\frac{z_{N}}{w_{N}} \leq$ $M^{2 P_{N}(c)}$ hold. Then the conclusions follow from the estimates $\frac{z_{N}}{v_{N}} \leq \frac{z_{N}}{w_{N}} \leq$ $M^{2 P_{N}(c)}$ and $\frac{v_{N}}{w_{N}} \leq \frac{z_{N}}{w_{N}} \leq M^{2 P_{N}(c)}$. This completes the proof.

For example $g_{t}=\frac{1}{m} f_{t}$ are the cases for $m \geq 1$, when $f_{t}$ is monotone increasing.

2.C.2 Dependence on initial values: Let $f_{t}: \mathbb{R}_{>0}^{n} \rightarrow(0, \infty)$ be a relative elementary function. Let us take two initial values:

$$
\bar{z}_{0}=\left(z_{0}, \ldots, z_{n-1}\right), \quad \bar{w}_{0}=\left(w_{0}, \ldots, w_{n-1}\right) \quad \in \mathbb{R}_{>0}^{n}
$$

and consider the corresponding orbits $\left\{z_{N}\right\}_{N=0}^{\infty}$ and $\left\{w_{N}\right\}_{N=0}^{\infty}$ defined by:

$$
z_{N}=f_{t}\left(z_{N-n}, \ldots, z_{N-1}\right), \quad w_{N}=f_{t}\left(w_{N-n}, \ldots, w_{N-1}\right), \quad(N \geq n)
$$

respectively. Let $\varphi$ and $\varphi_{t}$ be the functions corresponding to $f_{t}$.

Here we have more elaborate estimates:

Proposition 2.7. Let $f_{t}$ and the orbits $\left\{z_{N}\right\}_{N=0}^{\infty},\left\{w_{N}\right\}_{N=0}^{\infty}$ be as above with initial values $\bar{z}_{0}$ and $\bar{w}_{0}$. Then they satisfy uniformly bounded rates:

$$
\left(\frac{z_{N}}{w_{N}}\right)^{ \pm} \leq M^{2 P_{N}(c)}\left[\max _{0 \leq i \leq n-1}\left(\frac{z_{i}}{w_{i}}\right)^{ \pm 1}\right]^{c^{N}} \quad(N \geq n)
$$

where $c$ and $M$ are the Lipschitz constant and the number of the components for $\varphi$ respectively.

Proof: The idea of the proof is parallel to proposition 2.3 .

Let us put $x_{N}^{\prime}=\log _{t}\left(z_{N}\right)$ and $y_{N}^{\prime}=\log _{t}\left(w_{N}\right)$ respectively. Thus $\left\{x_{N}^{\prime}\right\}_{N}$ is the orbit for $\varphi_{t}$ with the initial value $x_{i}^{\prime}=\log _{t} z_{i}$ for $0 \leq i \leq n-1$, and similar for $\left\{y_{N}^{\prime}\right\}_{N}$.

Let $\left\{x_{N}\right\}_{N}$ be another orbit for $\varphi$ with the same initial value $x_{i}=\log _{t} z_{i}$ for $0 \leq i \leq n-1$, and similar for $\left\{y_{N}\right\}_{N}$.

Let $c \geq 1$ be the Lipschitz constant for $\varphi$. Let us estimate $\left|x_{N}-y_{N}\right|$ for $N \geq n$. Since $x_{n}=\varphi\left(x_{0}, \ldots, x_{n-1}\right)$ and $y_{n}=\varphi\left(y_{0}, \ldots, y_{n-1}\right)$, the estimate:

$$
\left|x_{n}-y_{n}\right|=\left|\varphi\left(x_{0}, \ldots, x_{n-1}\right)-\varphi\left(y_{0}, \ldots, y_{n-1}\right)\right| \leq c \max _{0 \leq i \leq n-1}\left|x_{i}-y_{i}\right|
$$


hold. Let us iterate the same estimates:

$$
\begin{aligned}
\left|x_{n+1}-y_{n+1}\right| & =\left|\varphi\left(x_{1}, \ldots, x_{n}\right)-\varphi\left(y_{1}, \ldots, y_{n}\right)\right| \\
& \leq c \max _{1 \leq i \leq n}\left|x_{i}-y_{i}\right| \leq c^{2} \max _{0 \leq i \leq n-1}\left|x_{i}-y_{i}\right| .
\end{aligned}
$$

The same process gives us the estimates:

$$
\left|x_{N}-y_{N}\right| \leq c^{N-n+1} \max _{0 \leq i \leq n-1}\left|x_{i}-y_{i}\right|=c^{N-n+1} \max _{0 \leq i \leq n-1} \log _{t}\left(\frac{z_{i}}{w_{i}}\right)^{ \pm 1} .
$$

On the other hand by lemma 2.4, the estimates:

$$
\left|x_{N}-x_{N}^{\prime}\right|,\left|y_{N}-y_{N}^{\prime}\right| \leq P_{N}(c) \log _{t} M
$$

hold, where $M$ is the number of the components for $\varphi$. So combining with these estimates, we have the followings:

$$
\begin{aligned}
& \max \left(\log _{t} \frac{z_{N}}{w_{N}}, \log _{t} \frac{w_{N}}{z_{N}}\right)=\left|\log _{t}\left(z_{N}\right)-\log _{t}\left(w_{N}\right)\right|=\left|x_{N}^{\prime}-y_{N}^{\prime}\right| \\
& \leq\left|x_{N}-x_{N}^{\prime}\right|+\left|y_{N}-y_{N}^{\prime}\right|+\left|x_{N}-y_{N}\right| \\
& \leq 2 P_{N}(c) \log _{t} M+c^{N} \max _{0 \leq i \leq n-1} \log _{t}\left(\frac{z_{i}}{w_{i}}\right)^{ \pm 1} \\
& \left.=\log _{t}\left\{M^{2 P_{N}(c)} \max _{0 \leq i \leq n-1}\left(\frac{z_{i}}{w_{i}}\right)^{ \pm 1}\right]^{c^{N}}\right\} .
\end{aligned}
$$

Thus one obtains the estimates:

$$
\left(\frac{z_{N}}{w_{N}}\right)^{ \pm 1} \leq M^{2 P_{N}(c)} \max _{0 \leq i \leq n-1}\left(\frac{z_{i}}{w_{i}}\right)^{ \pm c^{N}} .
$$

This completes the proof.

Now let $g_{t}$ and $f_{t}$ be two relatively elementary functions, and denote the corresponding pairs of the functions by $\left(\varphi, \varphi_{t}\right)$ and $\left(\psi, \psi_{t}\right)$ respectively. Let $\left(c_{f}, M_{f}\right)$ and $\left(c_{g}, M_{g}\right)$ be the Lipschitz constants and the numbers of the components for $\varphi$ and $\psi$ respectively.

Corollary 2.8. Let $g_{t}$ be tropically equivalent to $f_{t}$, and $\left\{z_{N}\right\}_{N}$ and $\left\{w_{N}\right\}_{N}$ be the orbits for $f_{t}$ and $g_{t}$ with the initial values $\bar{z}_{0}=\left(z_{0}, \ldots, z_{n-1}\right)$ and $\bar{w}_{0}=\left(w_{0}, \ldots, w_{n-1}\right)$ respectively. Then the estimates hold:

$$
\left(\frac{z_{N}}{w_{N}}\right)^{ \pm} \leq M^{4 P_{N}(c)}\left[\max _{0 \leq i \leq n-1}\left(\frac{z_{i}}{w_{i}}\right)^{ \pm 1}\right]^{c^{N}}
$$

where $c=\max \left(c_{f}, c_{g}\right)$ and $M=\max \left(M_{f}, M_{g}\right)$. 
Proof: Let $\left\{z_{N}^{\prime}\right\}_{N}$ be the orbit for $f_{t}$ with the initial value $\bar{w}_{0}=\left(w_{0}, \ldots, w_{n-1}\right)$. By proposition 2.7, one obtains the estimates:

$$
\left(\frac{z_{N}}{z_{N}^{\prime}}\right)^{ \pm 1} \leq M^{2 P_{N}(c)}\left[\max _{0 \leq i \leq n-1}\left(\frac{z_{i}}{w_{i}}\right)^{ \pm 1}\right]^{c^{N}} .
$$

On the other hand by proposition 2.3, one has another estimates:

$$
\left(\frac{z_{N}^{\prime}}{w_{N}}\right)^{ \pm 1} \leq M^{2 P_{N}(c)}
$$

By multiplying both sides, one obtains the desired estimates:

$$
\begin{aligned}
\left(\frac{z_{N}}{w_{N}}\right)^{ \pm 1} & =\left(\frac{z_{N}}{z_{N}^{\prime}}\right)^{ \pm 1}\left(\frac{z_{N}^{\prime}}{w_{N}}\right)^{ \pm 1} \leq M^{2 P_{N}(c)} M^{2 P_{N}(c)}\left[\max _{0 \leq i \leq n-1}\left(\frac{z_{i}}{w_{i}}\right)^{ \pm 1}\right]^{c^{N}} \\
& =M^{4 P_{N}(c)}\left[\max _{0 \leq i \leq n-1}\left(\frac{z_{i}}{w_{i}}\right)^{ \pm 1}\right]^{c^{N}} .
\end{aligned}
$$

This completes the proof.

Now we induce the main estimates:

Theorem 2.9. Let us take four relative elementary functions, $f_{t}, f_{t}^{\prime}, g_{t}, g_{t}^{\prime}$. Assume that they are all monotone increasing and all tropically equivalent. Let $\left\{v_{N}\right\}_{N}$ and $\left\{u_{N}\right\}_{N}$ be positive sequences which satisfy the estimates:

$$
\begin{aligned}
& f_{t}^{\prime}\left(v_{N-n}, \ldots, v_{N-1}\right) \leq v_{N} \leq f_{t}\left(v_{N-n}, \ldots, v_{N-1}\right), \\
& g_{t}^{\prime}\left(u_{N-n}, \ldots, u_{N-1}\right) \leq u_{N} \leq g_{t}\left(u_{N-n}, \ldots, u_{N-1}\right) .
\end{aligned}
$$

for all $N \geq n$. Then the ratios satisfy the uniform estimates:

$$
\left(\frac{v_{N}}{u_{N}}\right)^{ \pm 1} \leq M^{8 P_{N}(c)}\left[\max _{0 \leq i \leq n-1}\left(\frac{u_{i}}{v_{i}}\right)^{ \pm 1}\right]^{c^{N}}
$$

where $c=\max \left(c_{f}, c_{f^{\prime}}, c_{g}, c_{g^{\prime}}\right)$ and $M=\max \left(M_{f}, M_{f^{\prime}}, M_{g}, M_{g^{\prime}}\right)$.

Proof: Let us consider two orbits $\left\{z_{N}\right\}_{N}$ and $\left\{z_{N}^{\prime}\right\}_{N}$ defined by $z_{N}=$ $f_{t}\left(z_{N-n}, \ldots, z_{N-1}\right)$ and $z_{N}^{\prime}=f_{t}^{\prime}\left(z_{N-n}^{\prime}, \ldots, z_{N-1}^{\prime}\right)$ with the the same initial value $z_{i}=z_{i}^{\prime}=v_{i}$ for $0 \leq i \leq n-1$ respectively. Similarly by use of $g_{t}$ and $g_{t}^{\prime}$, one has orbits for $\left\{w_{N}\right\}_{N}$ and $\left\{w_{N}^{\prime}\right\}_{N}$ with the initial value $w_{i}=w_{i}^{\prime}=u_{i}$ for $0 \leq i \leq n-1$ respectively. 
Then by corollary 2.6, one has the estimates:

$$
\left(\frac{z_{N}}{v_{N}}\right)^{ \pm 1}, \quad\left(\frac{w_{N}}{u_{N}}\right)^{ \pm 1} \leq M^{2 P_{N}(c)} .
$$

On the other hand by corollary 2.8 , the estimates hold:

$$
\left(\frac{z_{N}}{w_{N}}\right)^{ \pm 1} \leq M^{4 P_{N}(c)}\left[\max _{0 \leq i \leq n-1}\left(\frac{z_{i}}{w_{i}}\right)^{ \pm 1}\right]^{c^{N}}=M^{4 P_{N}(c)}\left[\max _{0 \leq i \leq n-1}\left(\frac{v_{i}}{u_{i}}\right)^{ \pm 1}\right]^{c^{N}} .
$$

Thus from these two, one obtains the desired uniform estimates:

$$
\begin{aligned}
& \left(\frac{v_{N}}{u_{N}}\right)^{ \pm 1}=\left(\frac{v_{N}}{z_{N}}\right)^{ \pm 1}\left(\frac{z_{N}}{w_{N}}\right)^{ \pm 1}\left(\frac{w_{N}}{u_{N}}\right)^{ \pm 1} \\
& \leq M^{2 P_{N}(c)} M^{4 P_{N}(c)}\left[\max _{0 \leq i \leq n-1}\left(\frac{v_{i}}{u_{i}}\right)^{ \pm 1}\right]^{c^{N}} M^{2 P_{N}(c)} \\
& =M^{8 P_{N}(c)}\left[\max _{0 \leq i \leq n-1}\left(\frac{v_{i}}{u_{i}}\right)^{ \pm 1}\right]^{c^{N}} .
\end{aligned}
$$

This completes the proof.

2.D Evolutional dynamics: For simplicity of the notation, later on we will omit to denote the parameter $t$ for $f_{t}$ and just write $f$ for any relative elementary functions.

Let $f$ be a relative elementary function. A general equation of evolutional discrete dynamics is of the form:

$$
z_{N+1}^{t+1}=f\left(z_{N-l_{0}}^{t+1}, \ldots, z_{N}^{t+1}, z_{N-l_{1}}^{t}, \ldots, z_{N+k_{1}}^{t}, z_{N-l_{2}}^{t-1}, \ldots, z_{N+k_{2}}^{t-1}, \ldots, z_{N+k_{d+1}}^{t-d}\right)
$$

where $l_{i}, k_{j} \geq 0, N \geq \max \left(l_{0}, \ldots, l_{d+1}\right)$ and $t \geq d$, with initial values:

$$
\bar{z}_{0}^{0} \equiv\left\{z_{a}^{t}\right\}_{0 \leq a \leq \max \left(l_{0}, \ldots, l_{d+1}\right), t=0,1, \ldots} \cup\left\{z_{N}^{h}\right\}_{0 \leq h \leq d, N=0,1, \ldots} .
$$

As before one puts the Lipschitz constant and the number of the components by $c_{f}$ and $M_{f}$ for the corresponding (max, + )-function to $f$.

Let us put $l=\max \left(l_{0}, l_{1}, \ldots, l_{d+1}\right), k=\max \left(k_{1}, \ldots, k_{d+1}\right)$ and

$$
A(N, t) \equiv(t-d-1) k+N-l+n-1
$$

for $N \geq l+1$ and $t \geq d+1$.

Let us take $g$ tropically equivalent to $f$, and consider the dynamics $\left\{w_{n}^{t}\right\}$ defined by $g$ with any initial value $\bar{w}_{0}^{0}$. 
Now we put the initial rates by:

$$
\left[\bar{z}_{0}^{0}: \bar{w}_{0}^{0}\right] \equiv \sup _{0 \leq a \leq \max \left(l_{0}, \ldots, l_{d+1}\right), b=0,1, \ldots, \text { or } a=0,1, \ldots, 0 \leq b \leq d}\left\{\frac{z_{a}^{b}}{w_{a}^{b}}, \frac{w_{a}^{b}}{z_{a}^{b}}\right\} .
$$

Proposition 2.10. (1) Let $f$ and $g$ be tropically equivalent. Then any orbits $\left\{z_{N}^{t}\right\}_{N}$ and $\left\{w_{N}^{t}\right\}_{N}$ for $f$ and $g$ with the initial values $\bar{z}_{0}^{0}$ and $\bar{w}_{0}^{0}$ respectively, satisfy the estimates:

$$
\left(\frac{z_{N}^{t}}{w_{N}^{t}}\right)^{ \pm} \leq M^{4 P_{A(N, t)}(c)}\left[\bar{z}_{0}^{0}: \bar{w}_{0}^{0}\right]^{c^{A(N, t)}}
$$

where $c=\max \left(c_{f}, c_{g}\right)$ and $M=\max \left(M_{f}, M_{g}\right)$.

(2) Let $f, f^{\prime}, g, g^{\prime}$ be four relative elementary functions, and assume that they are all monotone increasing and all tropically equivalent. Let $\left\{v_{N}^{t}\right\}_{N, t}$ and $\left\{u_{N}^{t}\right\}_{N, t}$ be positive sequences so that these satisfy the estimates:

$$
\begin{aligned}
& f^{\prime}\left(v_{N-l_{0}}^{t+1}, \ldots, v_{N+k_{d+1}}^{t-d}\right) \leq v_{N+1}^{t+1} \leq f\left(v_{N-l_{0}}^{t+1}, \ldots, v_{N+k_{d+1}}^{t-d}\right), \\
& g^{\prime}\left(u_{N-l_{0}}^{t+1}, \ldots, u_{N+k_{d+1}}^{t-d}\right) \leq u_{N+1}^{t+1} \leq g\left(u_{N-l_{0}}^{t+1}, \ldots, u_{N+k_{d+1}}^{t-d}\right)
\end{aligned}
$$

for $N \geq l$ and $t \geq d$. Then the ratios satisfy the uniform estimates:

$$
\left(\frac{v_{N}^{t}}{u_{N}^{t}}\right)^{ \pm 1} \leq M^{8 P_{A(N, t)}(c)}\left[\bar{u}_{0}^{0}: \bar{v}_{0}^{0}\right]^{A^{A(N, t)}}
$$

for $N \geq l+1$ and $t \geq d+1$.

Proof: Let us check that in order to determine $z_{l+N}^{d+t}$, one has to iterate at most $(t-1) k+N$ times to apply $f$ for $N, t \geq 1$. Then the conclusions follow from corollary 2.8 and theorem 2.9 (see remark (1) below the proof of proposition 2.3).

Let us denote by $\alpha(N, t)$ the number of compositions of $f$ in order to determine $z_{N}^{t}$. It is an increasing function on both variables. We show the estimates $\alpha(l+N, d+t) \leq(t-1) k+N$.

Let $\Delta_{0}=\{(a, b) \in\{0,1, \ldots, k+l\} \times\{0,1, \ldots, d\} \cup\{0, \ldots, l\} \times\{d+1\}\}$ be the finite set. This is a basic building block in the sense that for $N, t \geq 1$, $z_{N+l}^{t+d}$ is determined if one knows $z_{N-1+a}^{t-1+b}$ for $(a, b) \in \Delta_{0}$.

We proceed by induction on $t . \alpha(l+N, d+1) \leq N$ clearly follows.

Suppose the conclusion follows for $t \leq t_{0}$, and so $\alpha\left(N+l, d+t_{0}\right) \leq$ $\left(t_{0}-1\right) k+N$ hold. Then $\alpha\left(l+1, d+t_{0}+1\right)=\alpha\left(l+k, d+t_{0}\right)+1 \leq$ 
$\left(t_{0}-1\right) k+k+1=t_{0} k+1$ hold. Next $\alpha\left(l+2, d+t_{0}+1\right)=\max (\alpha(l+$ $\left.\left.1, d+t_{0}+1\right), \alpha\left(l+k+1, d+t_{0}\right)\right)+1 \leq t_{0} k+2$. By use of the estimates $\alpha\left(N+l, d+t_{0}+1\right) \leq \max \left(\alpha\left(N-1+l, d+t_{0}+1\right), \alpha\left(N-1+l+k, d+t_{0}\right)\right)+1$, one can obtain the bounds $\alpha\left(N+l, d+t_{0}+1\right) \leq t_{0} k+N$.

This completes the proof.

\section{Asymptotic comparisons}

3.A Formal Taylor expansion and ODE: Let us consider a $C^{\alpha+1}$ function $u:(0, \infty) \rightarrow(0, \infty)$. Below we proceed to approximate $u$ very roughly by discrete dynamics defined by relative elementary functions of $n$ variables. For $1 \leq|i| \leq n-1$, let us take the Taylor expansions around $x \in(0, \infty)$ :

$$
u(x+i \epsilon)=u(x)+i \epsilon u_{x}+\frac{(i \epsilon)^{2}}{2} u_{2 x}+\cdots+\frac{(i \epsilon)^{\alpha}}{\alpha !} u_{\alpha x}+\frac{(i \epsilon)^{(\alpha+1)}}{(\alpha+1) !} u_{(\alpha+1) x}\left(\xi_{i}\right)
$$

for small $|\epsilon|<<1$, where:

$$
\begin{cases}x \leq \xi_{i} \leq x+i \epsilon, & i \geq 0 \\ x+i \epsilon \leq \xi_{i} \leq x & i<0\end{cases}
$$

(for our applications, we will choose $\alpha \leq 2$ later).

Let $f=\frac{k}{h}: \mathbb{R}_{>0}^{n} \rightarrow(0, \infty)$ be a relative elementary function, where $h$ and $k$ are both elementary. Later on we will assume positivity:

$$
h(\overline{0})>0 .
$$

Let us consider the discrete dynamics defined by $z_{N+1}=f\left(z_{N-n+1}, \ldots, z_{N}\right)$.

We put the fluctuation intervals by:

$$
I(n, \epsilon)=[-n \epsilon, 0] .
$$

For $N=0,1,2, \ldots$, let us put change of variables:

$$
z_{N} \equiv \epsilon u(\epsilon N)=\epsilon u(x), \quad\left(N=\frac{x}{\epsilon}\right) .
$$

Let us consider the difference:

$$
z_{N+1}-f\left(z_{N-n+1}, \ldots, z_{N}\right)=\epsilon u(x+\epsilon)-f(\epsilon u(x-(n-1) \epsilon), \ldots, \epsilon u(x))
$$


and insert the Taylor expansions:

$$
\begin{aligned}
=\epsilon(u+ & \left.\epsilon u_{x}+\frac{\epsilon^{2}}{2} u_{2 x}+\ldots\right) \\
& -f\left(\epsilon\left(u-(n-1) \epsilon u_{x}+\frac{(n-1)^{2} \epsilon^{2}}{2} u_{2 x}+\ldots\right), \ldots, \epsilon u\right) .
\end{aligned}
$$

By reordering the expansions with respect to the exponents of $\epsilon$, there are rational numbers $a_{0}, a_{1}, \cdots \in \mathbb{Q}$ so that the equality holds:

$$
\begin{aligned}
& \epsilon u(x+\epsilon)-f(\epsilon u(x-(n-1) \epsilon), \ldots, \epsilon u(x)) \\
& =\frac{\epsilon a_{0} u+\epsilon^{2} a_{1} u_{x}+\epsilon^{3} a_{2} u u_{x}+. .+\epsilon^{\alpha+1} a_{s} u_{\alpha x}+\epsilon^{\alpha+2} a_{s+1} u_{(\alpha+1) x}(\xi)+. .}{h(\epsilon u(x-(n-1) \epsilon), \ldots, \epsilon u(x))} \\
& \equiv \frac{\epsilon F^{1}(u)+\epsilon^{2} F^{2}\left(u_{x}\right)+\epsilon^{3} F^{3}\left(u, u_{x}\right)+. .+\epsilon^{m} F^{m^{\prime}}\left(u, . ., u_{(\alpha+1) x}(\xi)\right)+. .}{h(\epsilon u(x-(n-1) \epsilon), \ldots, \epsilon u(x))}
\end{aligned}
$$

where $F^{k}$ are monomials.

Let us choose finite subsets $A \subset\{1,2,3, \ldots\}$, and divide the expanded sum into two terms as:

$$
\begin{aligned}
& =\frac{\sum_{i \in A} \epsilon^{s_{i}} F^{s_{i}^{\prime}}\left(u, u_{x}, \ldots, u_{\alpha x}\right)}{h(\epsilon u(x-(n-1) \epsilon), \ldots)}+\frac{\Sigma_{j \in A^{c}} \epsilon^{s_{j}} F^{s_{j}^{\prime}}\left(u, u_{x}, \ldots, u_{(\alpha+1) x}(\xi)\right)}{h(\epsilon u(x-(n-1) \epsilon), . .)} \\
& \equiv \mathbf{F}\left(\epsilon, u, u_{x}, \ldots, u_{\alpha x}\right)+\epsilon^{2} \mathbf{F}^{1}\left(\epsilon, u, u_{x}, . ., u_{(\alpha+1) x}\left(\xi_{1}\right), . ., u_{(\alpha+1) x}\left(\xi_{n-1}\right)\right) .
\end{aligned}
$$

We always choose $A$ so that two conditions are satisfied;

(1) $\mathbf{F}$ do not contain $u_{(l+1) x}(\xi)$, and (2) $1 \in A$, i.e. $F^{1}$ is included in $\mathbf{F}$.

In all the concrete cases later, we choose relative elementary functions and $A$ so that the corresponding $F^{1}$ vanish.

Now fix $\epsilon>0$, and suppose $u$ obeys the equation:

$$
\mathbf{F}\left(\epsilon, u, u_{x}, \ldots, u_{\alpha x}\right)=0 \text {. }
$$

Then the difference satisfies the equality:

$$
\epsilon u(x+\epsilon)-f(\epsilon u(x-(n-1) \epsilon), \ldots, \epsilon u(x))=\epsilon^{2} \mathbf{F}^{1}\left(\epsilon, u, u_{x}, \ldots\right) .
$$

We say that $\mathbf{F}$ is the leading term, and $\mathbf{F}^{1}$ error one for $u$ respectively. 
Remark: Conversely when one starts from $\operatorname{ODE} \mathbf{F}\left(\epsilon, u, u_{x}, u_{\alpha x}\right)=0$, there will be several choices of relative elementary functions $f$ and $A$ with the leading term $\mathbf{F}$. Various choices of $f$ will assign different error terms $\mathbf{F}^{1}$, which reflect estimates of solutions $\mathbf{F}\left(\epsilon, u, u_{x}, \ldots, u_{\alpha x}\right)=0$. So 'better' choice of $f$ will give us 'better' estimates of large scale analysis of such solutions.

Let us define $\epsilon$ variation of $\mathbf{F}^{1}$ by

$$
\begin{aligned}
& \left\|\mathbf{F}^{1}\left(\epsilon, u, u_{x}, \ldots, u_{\alpha x}, u_{(\alpha+1) x}\left(\xi_{1}\right), \ldots, u_{(\alpha+1) x}\left(\xi_{n-1}\right)\right)\right\|_{\epsilon}(x) \equiv \\
& \sup _{\mu_{i}-x \in I(n, \epsilon)}\left|\mathbf{F}^{1}\left(\epsilon, u(x-\epsilon), . ., u_{\alpha x}(x-\epsilon), u_{(\alpha+1) x}\left(\mu_{1}\right), . ., u_{(\alpha+1) x}\left(\mu_{n-1}\right)\right)\right|
\end{aligned}
$$

where $I(n, \epsilon)$ is the fluctuation interval.

Let us say that a $C^{\alpha+1}$ function $u:(0, \infty) \rightarrow(0, \infty)$ is $\epsilon_{0}$ controlled, if there is some constant $C>0$ so that the $\epsilon_{0}$ variation of $\mathbf{F}^{1}$ satisfy the pointwise estimates for all $x \in(0, \infty)$ :

$$
C u(x) \geq\left\|\mathbf{F}^{1}\left(\epsilon, u, u_{x}, \ldots, u_{\alpha x}, u_{(\alpha+1) x}\left(\xi_{1}\right), \ldots, u_{(\alpha+1) x}\left(\xi_{n-1}\right)\right)\right\|_{\epsilon_{0}}(x)
$$

3.A.2 Comparison theorem for ODE: Let us take another relatively elementary function $g=\frac{d}{e}$ which is tropically equivalent to $f$. Let $v:(0, \infty) \rightarrow(0, \infty)$ be another $C^{\alpha+1}$ function. By replacing $f$ by $g$ and choosing another subsets $B \subset\{1,2,3, \ldots\}$ in $3 . A$, one has its leading and error terms $\mathbf{G}$ and $\mathbf{G}^{1}$ respectively. Then we have the equalities:

$$
\begin{aligned}
& \epsilon v(x+\epsilon)-g(\epsilon v(x-(n-1) \epsilon), \ldots, \epsilon v(x)) \\
& =\frac{\sum_{i \in B} \epsilon^{s_{i}} G^{s_{i}^{\prime}}\left(v, v_{x}, \ldots, v_{\alpha x}\right)}{e(\epsilon v(x-(n-1) \epsilon), \ldots)}+\frac{\sum_{j \in B^{c}} \epsilon^{s_{j}} G^{s^{\prime}}\left(v, v_{x}, \ldots, v_{(\alpha+1) x}\left(\xi^{\prime}\right)\right)}{e(\epsilon v(x-(n-1) \epsilon), \ldots)} \\
& \equiv \mathbf{G}\left(\epsilon, v, \ldots, v_{\alpha x}\right)+\epsilon^{2} \mathbf{G}^{1}\left(\epsilon, v, \ldots, v_{\alpha x}, v_{(\alpha+1) x}\left(\xi_{1}^{\prime}\right), \ldots, v_{(\alpha+1) x}\left(\xi_{n-1}^{\prime}\right)\right) .
\end{aligned}
$$

Let us fix a small $\epsilon>0$, and take two positive solutions $u, v:(0, \infty) \rightarrow$ $(0, \infty)$ to the equations:

$$
\mathbf{F}\left(\epsilon, u, u_{x}, \ldots\right)=0, \quad \mathbf{G}\left(\epsilon, v, v_{x}, \ldots\right)=0 .
$$

Now we compare their ratios:

$$
\left(\frac{u(x)}{v(x)}\right)^{ \pm 1}=\left\{\frac{u(x)}{v(x)}, \frac{v(x)}{u(x)}\right\} .
$$


For this we introduce the initial rates:

$$
[u: v]_{\epsilon} \equiv \sup _{x \in(0, \epsilon]}\left(\frac{u(x)}{v(x)}\right)^{ \pm 1} .
$$

Recall that associated with $f$ are the Lipschitz constant $c_{f} \geq 1$ and the number of the components $M_{f}$. Let us put $c=\max \left(c_{f}, c_{g}\right)$ and $M=$ $\max \left(M_{f}, M_{g}\right)$.

Theorem 3.1. Let $f$ and $g$ be both relatively elementary and increasing functions of $n$ variables, which are mutually tropically equivalent. Let $\mathbf{F}$ and $\mathbf{G}$ be their leading terms of order at most $\alpha \geq 0$, and take positive $C^{\alpha+1}$ solutions $u, v:(0, \infty) \rightarrow(0, \infty)$ to the equations:

$$
\mathbf{F}\left(\epsilon, u, u_{x}, \ldots, u_{\alpha x}\right)=0, \quad \mathbf{G}\left(\epsilon, v, v_{x}, \ldots, v_{\alpha x}\right)=0 .
$$

Assume both $u$ and $v$ are $\epsilon_{0}$ controlled bounded by $C$. Then for any $0<\epsilon \leq \min \left(\frac{1}{2 C}, \epsilon_{0}\right)$, the estimates hold:

$$
\left(\frac{u(x)}{v(x)}\right)^{ \pm 1} \leq(2 M)^{8{\frac{\epsilon^{-1} x+1}{c-1}}_{c-1}}[u: v]_{n \epsilon}^{c^{\epsilon^{-1} x+1}} .
$$

Proof: Let $f$ and $g$ be both $n$ variables, and $\left(\mathbf{F}, \mathbf{F}^{1}\right)$ and $\left(\mathbf{G}, \mathbf{G}^{1}\right)$ be pairs of leading and error terms respectively.

Let us choose $0<\epsilon \leq \min \left(\frac{1}{2 C}, \epsilon_{0}\right)$. By the assumption, the pointwise estimates hold:

$$
C u(x+\epsilon) \geq\left|\mathbf{F}^{1}\left(\epsilon, u(x), u_{x}(x), \ldots, u_{\alpha x}(x), u_{(\alpha+1) x}\left(\xi_{1}\right), \ldots, u_{(\alpha+1) x}\left(\xi_{n-1}\right)\right)\right| .
$$

In particular the estimates $\epsilon^{2}\left|\mathbf{F}^{1}\right| \leq \frac{1}{2 C} \epsilon\left|\mathbf{F}^{1}\right| \leq \frac{1}{2} \epsilon u(x+\epsilon)$ hold.

Let us consider the equalities:

$$
\begin{aligned}
& \epsilon u(x+\epsilon)-f(\epsilon u(x-(n-1) \epsilon), \ldots, \epsilon u(x)) \\
& =\mathbf{F}\left(\epsilon, u, \ldots, u_{\alpha x}\right)+\epsilon^{2} \mathbf{F}^{1}\left(\epsilon, u, \ldots, u_{\alpha x}, u_{(\alpha+1) x}\left(\xi_{1}\right), \ldots, u_{(\alpha+1) x}\left(\xi_{n-1}\right)\right) \\
& =\epsilon^{2} \mathbf{F}^{1}\left(\epsilon, u, u_{x}, \ldots, u_{\alpha x}, u_{(\alpha+1) x}\left(\xi_{1}\right), \ldots, u_{(\alpha+1) x}\left(\xi_{n-1}\right)\right)
\end{aligned}
$$

since $u$ obeys the equation $\mathbf{F}\left(\epsilon, u, u_{x}, \ldots\right)=0$.

Then combining with the above inequality, one obtains the estimates:

$$
\begin{aligned}
\frac{1}{2} f(\epsilon u(x-(n-1) \epsilon), & \ldots, \epsilon u(x)) \leq \epsilon u(x+\epsilon) \\
& \leq 2 f(\epsilon u(x-(n-1) \epsilon), \ldots, \epsilon u(x)) .
\end{aligned}
$$


By the same way one obtains the estimates by replacing $f$ by $g$ :

$$
\begin{aligned}
\frac{1}{2} g(\epsilon v(x-(n-1) \epsilon), & \ldots, \epsilon v(x)) \leq \epsilon v(x+\epsilon) \\
& \leq 2 g(\epsilon v(x-(n-1) \epsilon), \ldots, \epsilon v(x)) .
\end{aligned}
$$

$f, \frac{1}{2} f$ and $2 f$ are tropically equivalent, and $\frac{1}{2} f, 2 f, \frac{1}{2} g, 2 g$ are all so by the assumption. Notice that the number of the components for $\frac{1}{2} f$ and $2 f$ are both $2 M_{f}$.

Thus the estimates hold by theorem 2.9:

$$
\left(\frac{u(N \epsilon)}{v(N \epsilon)}\right)^{ \pm 1} \leq(2 M)^{8 P_{N}(c)} \sup _{0 \leq i \leq n-1}\left(\frac{u(\epsilon i)}{v(\epsilon i)}\right)^{ \pm c^{N}} \leq(2 M)^{8 P_{N}(c)}\left([u: v]_{(n-1) \epsilon}\right)^{c^{N}} .
$$

For any $0 \leq \mu \leq \epsilon$, let us apply the above estimates for the translations $u(x+\mu)$ and $v(x+\mu)$. Then one obtains the estimates:

$$
\begin{aligned}
& \left(\frac{u(N \epsilon+\mu)}{v(N \epsilon+\mu)}\right)^{ \pm 1} \leq(2 M)^{8 P_{N}(c)}[u: v]_{n \epsilon}^{c^{N}} \\
& =(2 M)^{8 \frac{c^{N-n+1}-1}{c-1}}[u: v]_{n \epsilon}^{c^{N}} \leq(2 M)^{8 \frac{\epsilon^{\epsilon^{-1}(N \epsilon+\mu)-n+2}-1}{c-1}}[u: v]_{n \epsilon}^{\epsilon^{-1}(N \epsilon+\mu)+1}
\end{aligned}
$$

since $P_{N}(c)=\frac{c^{N-n+1}-1}{c-1}$.

Such $N \epsilon+\mu$ takes all the points $x \in(0, \infty)$, and so the estimates hold:

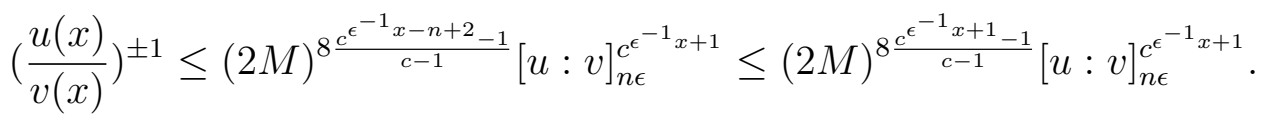

This completes the proof.

Example: Let us consider a simple equation:

$$
\mathbf{F}\left(u, u_{x}\right)=u_{x}+u^{2}=0 .
$$

It has solutions $u(x)=\frac{a}{1+a x}$ with the initial values $u(0)=a>0$. Let us put $z_{N}=\epsilon u(x)$ with $x=N \epsilon$ and take the Taylor expansion $\epsilon u(x+$ $\epsilon)=\epsilon u(x)+\epsilon^{2} u_{x}(x)+\frac{\epsilon^{3}}{2} u_{2 x}(\xi)$. We choose the relative elementary function $f(x)=x(1+x)^{-1}$ and calculate the difference:

$$
\begin{aligned}
& u(x+\epsilon)-f(\epsilon u(x)) \\
& =\epsilon^{2} \frac{u_{x}+u^{2}}{1+\epsilon u}(x)+\epsilon^{3} \frac{\frac{1}{2} u_{2 x}(\xi)+u(x) u_{x}(x)+\frac{\epsilon}{2} u(x) u_{2 x}(\xi)}{1+\epsilon u(x)}
\end{aligned}
$$


Thus $u$ is $\epsilon_{0}$ controlled, since $1+\epsilon u(x) \geq 1$, and the estimates $\left|u_{2 x}(\xi)\right|,\left|u u_{x}(x)\right|$, $\left|u(x) u_{2 x}(\xi)\right| \leq C u(x)$ hold uniformly in $x \in(0, \infty)$ for some $C=C\left(a, \epsilon_{0}\right) \geq 0$ and $|x-\xi| \leq \epsilon$.

The corresponding $(\max ,+)$ function to $f$ is given by $V_{N+1}=V_{N}-$ $\max \left(0, V_{N}\right)$. Notice the equality $V_{N}-\max \left(0, V_{N}\right)=V_{N}-\max \left(0, V_{N}, V_{N}\right)$. The tropical inverse for the latter is given by $g(y)=y(1+2 y)^{-1}$. By choosing the same scaling parameter, one obtains the leading term $\mathbf{G}\left(v, v_{x}\right)=v_{x}+2 v^{2}$. It has solutions $v(x)=\frac{a^{\prime}}{2 a^{\prime} x+1}$, and the ratio is in fact uniformly bounded:

$$
\left(\frac{u(x)}{v(x)}\right)^{ \pm}=\left(\frac{a\left(2 a^{\prime} x+1\right)}{a^{\prime}(a x+1)}\right)^{ \pm 1} \leq 2\left(\frac{a}{a^{\prime}}\right)^{ \pm 1} \leq 2[u: v]_{\epsilon} .
$$

3.B Evolutional dynamics: Here we treat partial differential equations. The process of $3 . B$ is quite parallel to $3 . A$ by introducing time parameter.

A general equation of evolutional discrete dynamics is of the form:

$$
z_{N+1}^{t+1}=f\left(z_{N-l_{0}}^{t+1}, \ldots, z_{N}^{t+1}, z_{N-l_{1}}^{t}, \ldots, z_{N+k_{1}}^{t}, z_{N-l_{2}}^{t-1}, \ldots, z_{N+k_{2}}^{t-1}, \ldots, z_{N+k_{d+1}}^{t-d}\right)
$$

where $l_{i}, k_{j} \geq 0, N \geq l \equiv \max \left(l_{0}, \ldots, l_{d+1}\right)$ and $t \geq d$, with initial values:

$$
\bar{z}_{0}^{0} \equiv\left\{z_{a}^{t}\right\}_{0 \leq a \leq l, t=0,1, \ldots} \cup\left\{z_{N}^{h}\right\}_{0 \leq h \leq d, N=0,1, \ldots} .
$$

Now let us consider a $C^{\alpha+1}$ function $u:(0, \infty) \times\left[0, T_{0}\right) \rightarrow(0, \infty)$, and introduce another parameters by

$$
N=\frac{x}{\epsilon^{p}}, \quad t=\frac{s}{\epsilon^{q}}, \quad \epsilon^{m} u(x, s)=z_{N}^{t}
$$

where $\epsilon>0$ is a small constant, and $p, q \geq 1, m \geq 0$ are integers. Then we take the Taylor expansions:

$$
\begin{aligned}
& u\left(x+i \epsilon^{p}, s+j \epsilon^{q}\right)=u+i \epsilon^{p} u_{x}+j \epsilon^{q} u_{s}+\frac{\left(i \epsilon^{p}\right)^{2}}{2} u_{2 x}+\frac{\left(j \epsilon^{q}\right)^{2}}{2} u_{2 s} \\
& +j \epsilon^{q} i \epsilon^{p} u_{x s}+\cdots+\frac{\left(i \epsilon^{p}\right)^{\alpha}}{\alpha !} u_{\alpha x}+\frac{\left(j \epsilon^{q}\right)^{\alpha}}{\alpha !} u_{\alpha s} \\
& +\frac{\left(i \epsilon^{p}\right)^{(\alpha+1)}}{(\alpha+1) !} u_{(\alpha+1) x}\left(\xi_{i j}\right)+\cdots+\frac{\left(j \epsilon^{q}\right)^{(\alpha+1)}}{(\alpha+1) !} u_{(\alpha+1) s}\left(\xi_{i j}\right) \\
& \equiv u+i \epsilon^{p} u_{x}+j \epsilon^{q} u_{s}+\frac{\left(i \epsilon^{p}\right)^{2}}{2} u_{2 x}+\frac{\left(j \epsilon^{q}\right)^{2}}{2} u_{2 s}+j \epsilon^{q} i \epsilon^{p} u_{x s} \\
& \quad+\cdots+\frac{\left(i \epsilon^{p}\right)^{\alpha}}{\alpha !} u_{\alpha x}+\frac{\left(j \epsilon^{q}\right)^{\alpha}}{\alpha !} u_{\alpha s}+\Sigma_{\bar{a}} \frac{\left(i \epsilon^{p}\right)^{a}\left(j \epsilon^{q}\right)^{\alpha+1-a}}{(\alpha+1) !} u_{\bar{a}}\left(\xi_{i j}\right)
\end{aligned}
$$


where $\bar{a}=\left(y_{i_{1}}, \ldots, y_{i_{\alpha+1}}\right), y_{j}=x$ or $s$, and $\left|(x, s)-\xi_{i j}\right| \leq\left|\left(i \epsilon^{p}, j \epsilon^{q}\right)\right|$.

Let $f=\frac{k}{h}: \mathbb{R}_{>0}^{n} \rightarrow(0, \infty)$ be a relative elementary function, and consider the difference as in $3 . A$ :

$$
\begin{aligned}
& z_{N+1}^{t+1}-f\left(z_{N-l_{0}}^{t+1}, \ldots, z_{N}^{t+1}, z_{N-l_{1}}^{t}, \ldots, z_{N+k_{1}}^{t}, \ldots, z_{N+k_{d+1}}^{t-d}\right) \\
& =\epsilon^{m} u\left(x+\epsilon^{p}, s+\epsilon^{q}\right) \\
& \quad-f\left(\epsilon^{m} u\left(x-l_{0} \epsilon^{p}, s+\epsilon^{q}\right), \ldots, \epsilon^{m} u\left(x+k_{d+1} \epsilon^{p}, s-d \epsilon^{q}\right)\right) .
\end{aligned}
$$

By reordering the expansions with respect to the exponents of $\epsilon$, there are rational numbers $a_{0}, a_{1}, \cdots \in \mathbb{Q}$ so that the above difference is equal to the following:

$$
\begin{aligned}
& \epsilon^{m} \frac{a_{0} u+\epsilon^{p} a_{1} u_{x}+\epsilon^{q} a_{2} u_{s}+\epsilon^{m+p} a_{3} u u_{x}+. .+\left(i \epsilon^{p}\right)^{h}\left(j \epsilon^{q}\right)^{\alpha+1-h} a_{h} u_{\bar{h}}\left(\xi_{i j}\right)+. .}{h\left(\epsilon^{m} u\left(x-l_{0} \epsilon^{p}, s+\epsilon^{q}\right), \ldots, \epsilon^{m} u\left(x+k_{d+1} \epsilon^{p}, s-d \epsilon^{q}\right)\right)} \\
& \equiv \frac{\epsilon^{m} F^{1}(u)+\epsilon^{m+p} F^{2}\left(u_{x}\right)+\epsilon^{m+q} F^{3}\left(u_{s}\right)+\epsilon^{2 m+p} F^{4}\left(u, u_{x}\right)+\ldots}{h\left(\epsilon^{m} u\left(x-l_{0} \epsilon^{p}, s+\epsilon^{q}\right), \ldots, \epsilon^{m} u\left(x+k_{d+1} \epsilon^{p}, s-d \epsilon^{q}\right)\right)}
\end{aligned}
$$

where $F^{k}$ are monomials.

Let us choose finite subsets $A \subset\{1,2,3, \ldots\}$, and divide the expanded sum into two terms as:

$$
\begin{aligned}
& \epsilon^{m} u\left(x+\epsilon^{p}, s+\epsilon^{q}\right) \\
&-f\left(\epsilon^{m} u\left(x-l_{0} \epsilon^{p}, s+\epsilon^{q}\right), \ldots, \epsilon^{m} u\left(x+k_{d+1} \epsilon^{p}, s-d \epsilon^{q}\right)\right) \\
&= \frac{\epsilon^{m} F^{1}(u)+\epsilon^{m+p} F^{2}\left(u_{x}\right)+\epsilon^{m+q} F^{3}\left(u_{s}\right)+\epsilon^{2 m+p} F^{4}\left(u, u_{x}\right)+\ldots}{h\left(\epsilon^{m} u\left(x-l_{0} \epsilon^{p}, s+\epsilon^{q}\right), \ldots, \epsilon^{m} u\left(x+k_{d+1} \epsilon^{p}, s-d \epsilon^{q}\right)\right)} \\
&= \frac{\sum_{i \in A} \epsilon^{s_{i}} F^{s^{\prime}}\left(u, u_{x}, u_{s}, \ldots, u_{\alpha s}\right)+\Sigma_{j \in A^{c}} \epsilon^{s_{j}} F^{s_{j}^{\prime}}\left(u, u_{x}, \ldots, u_{\bar{a}}\left(\xi_{i j}\right)\right)}{h\left(\epsilon^{m} u\left(x-l_{0} \epsilon^{p}, s+\epsilon^{q}\right), \ldots, \epsilon^{m} u\left(x+k_{d+1} \epsilon^{p}, s-d \epsilon^{q}\right)\right)} \\
& \equiv \mathbf{F}\left(\epsilon, u, u_{x}, u_{s}, \ldots, u_{\alpha s}\right)+\epsilon^{m+1} \mathbf{F}^{1}\left(\epsilon, u, u_{x}, \ldots,\left\{u_{\bar{a}}\left(\xi_{i j}\right)\right\}_{\bar{a}, i, j}\right) .
\end{aligned}
$$

As in $3 . A$, we always choose $A$ so that $\mathbf{F}$ do not contain $u_{\bar{a}}(\xi)$ and $1 \in A$. We call $\mathbf{F}$ as the leading term and $\mathbf{F}^{1}$ the error term respectively.

3.B.2 $\epsilon$-controlledness: Now we return to the starting point. Let $f$ be a relative elementary function, and consider the discrete dynamics $z_{N+1}^{t+1}=$ $f\left(z_{N-l_{0}}^{t+1}, \ldots, z_{N}^{t+1}, z_{N-l_{1}}^{t}, \ldots,, z_{N+k_{d+1}}^{t-d}\right)$. After one chooses integers $p, q, m$ for change of variables, one determines the leading and error terms $\mathbf{F}$ and $\mathbf{F}^{1}$ respectively. 
For $(a, b) \in \mathbb{Z}^{2}$, let $L(a, b)=\{(t a, t b): t \in[0,1]\} \subset \mathbb{R}^{2}$ be the segment. Then for the set:

$$
\begin{aligned}
D \equiv & \left\{(1,1),\left(-l_{0}, 1\right), \ldots,(0,1),\left(-l_{1}, 0\right), \ldots,\left(k_{1}, 0\right)\right. \\
& \left.\left(-l_{2},-1\right), \ldots,\left(k_{2},-1\right), \ldots,\left(-l_{d+1},-d\right), \ldots,\left(k_{d+1},-d\right)\right\}
\end{aligned}
$$

we put the fluctuation domain as:

$$
D(\epsilon, p, q)=\left\{\left(L\left(\epsilon^{p} a, \epsilon^{q} b\right):(a, b) \in D\right\} \subset \mathbb{R}^{2} .\right.
$$

For example $D=\{(1,1),(2,0),(-1,1)\}$ for $z_{N+1}^{t+1}=f\left(z_{N}^{t}, z_{N+2}^{t}, z_{N-1}^{t+1}\right)$.

Let us regard $\mathbf{F}^{1}$ as a function on the variables $\left(x, s,\left\{\xi_{i j}\right\}_{i, j}\right)$. Then we define its $\epsilon$ variation:

$$
\begin{aligned}
& \left\|\mathbf{F}^{1}\right\|_{\epsilon}(x, s) \equiv \sup _{\xi_{i j}-\left(x-\epsilon^{p}, s-\epsilon^{q}\right) \in D(\epsilon, p, q)} \mid \mathbf{F}^{1}\left(\epsilon, u\left(x-\epsilon^{p}, s-\epsilon^{q}\right)\right. \\
& \left.u_{x}\left(x-\epsilon^{p}, s-\epsilon^{q}\right), u_{s}\left(x-\epsilon^{p}, s-\epsilon^{q}\right), \ldots, u_{\alpha s}\left(x-\epsilon^{p}, s-\epsilon^{q}\right),\left\{u_{\bar{a}}\left(\xi_{i j}\right)\right\}\right) \mid .
\end{aligned}
$$

Let $u:(0, \infty) \times\left[0, T_{0}\right) \rightarrow(0, \infty)$ be a $C^{\alpha+1}$ function.

Definition 3.1. $u$ is $\epsilon_{0}$ controlled bounded by $C$, if $\epsilon_{0}$ variation of $\mathbf{F}^{1}$ satisfies the pointwise estimates:

$$
C u(x, s) \geq\left\|\mathbf{F}^{1}\right\|_{\epsilon_{0}}(x, s)
$$

for all $(x, s) \in(0, \infty) \times\left[0, T_{0}\right)$.

3.B.2.2 Higher derivative rates: Let $u:(0, \infty) \times\left[0, T_{0}\right) \rightarrow(0, \infty)$ be a $C^{\alpha+1}$ function and $f$ be a relative elementary function. let us consider the expansions of the differences in 3.B:

$$
\begin{aligned}
& \epsilon^{m} u\left(x+\epsilon^{p}, s+\epsilon^{q}\right) \\
& \quad \quad-f\left(\epsilon^{m} u\left(x-l_{0} \epsilon^{p}, s+\epsilon^{q}\right), \ldots, \epsilon^{m} u\left(x+k_{d+1} \epsilon^{p}, s-d \epsilon^{q}\right)\right) \\
& =\mathbf{F}\left(\epsilon, u, u_{x}, u_{s}, \ldots, u_{\alpha s}\right)+\epsilon^{m+1} \mathbf{F}^{1}\left(\epsilon, u, u_{x}, \ldots,\left\{u_{\bar{a}}\left(\xi_{i j}\right)\right\}_{\bar{a}, i, j}\right) .
\end{aligned}
$$

$\mathbf{F}$ has order at most $\alpha$, while $\mathbf{F}^{1}$ may contain derivatives of $u$ smaller than $\alpha+1$ in general.

Let us say that the error term $\mathbf{F}^{1}$ is admissible, if it is of the form:

$$
\mathbf{F}^{1}=\Sigma_{a \in A^{c}} c_{a} \epsilon^{s_{a}} H_{a}\left(\epsilon^{m} u\left(x-l_{0} \epsilon^{p}, s+\epsilon^{q}\right), \ldots\right) u_{\bar{a}}\left(\xi_{i j}\right)
$$


where (1) $|\bar{a}|=\alpha+1$ and $(2)\left\|H_{a}\left(x_{1}, x_{2}, \ldots\right)\right\|_{C^{0}} \leq 1$ for any $x_{1}, x_{2} \cdots \geq 0$.

For this case we put the error constants by:

$$
C_{\mathbf{F}^{1}} \equiv \Sigma_{a \in A^{c}}\left|c_{a}\right| \in \mathbb{Q}_{>0} .
$$

The error constants are determined by the coefficients of rational functions $f$ and of the Taylor expansions. Our applications later are all admissible cases.

Let us introduce variation of order $\alpha+1$ of $u$ by:

$$
\|u\|_{\alpha+1}(x, s)=\max _{\partial_{i}=\partial_{x}, \partial_{s}}\left\{\sup _{\xi-\left(x-\epsilon^{p}, s-\epsilon^{q}\right) \in D(\epsilon, p, q)}\left|\frac{\partial^{\alpha+1} u}{\partial_{1} \ldots \partial_{\alpha+1}}\right|(\xi)\right\} .
$$

Let us say that $u$ satisfies uniform $\epsilon$ variation, if there is a constant $C$ so that it satisfies the estimates:

$$
C u(x, s) \geq\|u\|_{\alpha+1}(x, s)
$$

for all $(x, s) \in(0, \infty) \times\left[0, T_{0}\right)$.

We put the variation constant by:

$$
V(u) \equiv \sup _{(x, s) \in(0, \infty) \times\left[0, T_{0}\right)} \frac{\|u\|_{\alpha+1}(x, s)}{u(x, s)} .
$$

Lemma 3.2. Suppose $\mathbf{F}^{1}$ is admissible, and $u$ satisfies uniform $\epsilon$ variation bounded by $C$. Then $u$ is $\epsilon$ controlled bounded by $C C_{\mathbf{F}^{1}}$.

Proof: By admissibility, the estimates hold:

$$
\begin{aligned}
\left\|\mathbf{F}^{1}\right\|_{\epsilon}(x, s) & \leq \Sigma_{a \in A^{c}}\left|c_{a}\right| \epsilon^{s_{a}}\left|H_{a}\left(\epsilon^{m} u\left(x-l_{0} \epsilon^{p}, s+\epsilon^{q}\right), \ldots\right)\right|\|u\|_{\alpha+1}(x, s) \\
& \leq \Sigma_{a \in A^{c}}\left|c_{a}\right|\|u\|_{\alpha+1}(x, s) \leq C_{\mathbf{F}^{1}}\|u\|_{\alpha+1}(x, s) \leq C_{\mathbf{F}^{1}} C u(x, s) .
\end{aligned}
$$

Let $u:(0, \infty) \times\left[0, T_{0}\right) \rightarrow(0, \infty)$ be a $C^{\alpha+1}$ function. Here we consider classes of functions which satisfy uniform rates between higher derivatives and lowest values.

Let us introduce the derivative constants of $\alpha+1$, which is given by:

$$
\|u\|_{\alpha+1}=\max _{\partial_{i}=\partial_{x}, \partial_{s}}\left\{\left\|\frac{\partial^{\alpha+1} u}{\partial_{1} \ldots \partial_{\alpha+1}}\right\|_{C^{0}\left((0, \infty) \times\left[0, T_{0}\right)\right)}\right\} .
$$

Suppose $u$ satisfies two conditions: 
(1) $\|u\|_{\alpha+1}<\infty$ is finite and (2) $c=\inf _{(x, s) \in(0, \infty) \times\left[0, T_{0}\right)} u(x, s)>0$ is positive. Then we say that the ratio:

$$
K(u) \equiv \frac{\|u\|_{\alpha+1}}{c}
$$

is the derivative rates of order $\alpha+1$. In general the estimates hold:

$$
V(u) \leq K(u) .
$$

Now we state the following which requires more practical conditions:

Lemma 3.3. Suppose $\mathbf{F}^{1}$ is admissible, and the derivative rates of order $\alpha+1$ is of finite, $K(u)<\infty$. Then for any $\epsilon>0, u$ is $\epsilon$ controlled bounded by $C_{\mathbf{F}^{1}} K(u)$.

Proof: By the conditions, the error term $\mathbf{F}^{1}$ satisfies the estimates:

$$
\begin{aligned}
\left\|\mathbf{F}^{1}\right\|_{\epsilon}(x, s) & \leq C_{\mathbf{F}^{1}}\|u\|_{\alpha+1}(x, s) \leq C_{\mathbf{F}^{1}}\|u\|_{\alpha+1} \\
& =C_{\mathbf{F}^{1}} K(u) c \leq C_{\mathbf{F}^{1}} K(u) u(x, s) .
\end{aligned}
$$

So $u$ is $\epsilon$-controlled bounded by $C_{\mathbf{F}^{1}} K(u)$ for any $\epsilon>0$.

This completes the proof.

3.B.3 Comparison theorem: Let $g$ be tropically equivalent to $f$, and choose the same scaling parameters. By replacing $f$ by $g$ above, one obtains another leading and error terms $\mathbf{G}$ and $\mathbf{G}^{1}$ with the equalities:

$$
\begin{aligned}
& \epsilon^{m} v\left(x+\epsilon^{p}, s+\epsilon^{q}\right)- \\
& \quad g\left(\epsilon^{m} v\left(x-l_{0} \epsilon^{p}, s+\epsilon^{q}\right), \ldots, \epsilon^{m} v\left(x+k_{d+1} \epsilon^{p}, s-d \epsilon^{q}\right)\right) \\
& =\mathbf{G}\left(\epsilon, v, v_{x}, v_{s}, \ldots, v_{\alpha s}\right)+\epsilon^{m+1} \mathbf{G}^{1}\left(\epsilon, v, v_{x}, v_{s}, \ldots, v_{\alpha s},\left\{v_{\bar{a}}\left(\eta_{i j}\right)\right\}_{\bar{a}, i, j}\right) .
\end{aligned}
$$

Let us fix a small $\epsilon>0$, and take two positive solutions $u, v:(0, \infty) \times$ $\left[0, T_{0}\right) \rightarrow(0, \infty)$ to the corresponding PDEs:

$$
\mathbf{F}\left(\epsilon, u, u_{x}, u_{s}, \ldots, u_{\alpha x}, u_{\alpha s}\right)=0, \quad \mathbf{G}\left(\epsilon, v, v_{x}, v_{s}, \ldots, v_{\alpha x}, v_{\alpha s}\right)=0 .
$$

In order to estimate their ratios $\left(\frac{u(x, s)}{v(x, s)}\right)^{ \pm 1}$, we introduce the initial rates:

$$
[u: v]_{\epsilon} \equiv \sup _{(x, s) \in(0, \infty) \times\left[0, \epsilon^{q}\right] \cup\left(0, \epsilon^{p}\right] \times\left[0, T_{0}\right)}\left(\frac{u(x, s)}{v(x, s)}\right)^{ \pm 1} .
$$

Recall the Lipschitz constant $c_{f}$ and the number of the components $M_{f}$ for $f$. Let us put $c=\max \left(c_{f}, c_{g}\right), M=\max \left(M_{f}, M_{g}\right), k=\max \left(k_{1}, \ldots, k_{d+1}\right)$ and $L=\max (l, d)$ for $l=\max \left(l_{0}, l_{1}, \ldots, l_{d+1}\right)$. 
Corollary 3.4. Let $f$ and $g$ be both relatively elementary and increasing functions of $n$ variables, which are mutually tropically equivalent. Let $\mathbf{F}$ and $\mathbf{G}$ be their leading terms of order at most $\alpha \geq 0$, and take positive $C^{\alpha+1}$ solutions $u, v:(0, \infty) \times\left[0, T_{0}\right) \rightarrow(0, \infty)$ to the equations:

$$
\mathbf{F}\left(\epsilon, u, u_{x}, u_{s}, \ldots, u_{\alpha x}, u_{\alpha s}\right)=0, \mathbf{G}\left(\epsilon, v, v_{x}, v_{s}, \ldots, v_{\alpha x}, v_{\alpha s}\right)=0 .
$$

Assume both $u$ and $v$ are $\epsilon_{0}$ controlled bounded by $C$. Then for any $0<\epsilon \leq \min \left(\frac{1}{2 C}, \epsilon_{0}\right)$ and $D=\max (p, q)$, the estimates hold:

$$
\left(\frac{u(x, s)}{v(x, s)}\right)^{ \pm 1} \leq(2 M)^{8 \frac{\epsilon^{\epsilon^{-D}(x+k s)+1}-1}{c-1}}[u: v]_{(L+1) \epsilon}^{\epsilon^{-D}(x+k s)+n} .
$$

Proof: Recall $A(N, t)=(t-d-1) k+N-l+n-1$ for $N \geq l+1$ and $t \geq d+1$. Let us take $0<\epsilon \leq \min \left(\frac{1}{2 C}, \epsilon_{0}\right)$. Combining with proposition 2.10, the parallel argument to the proof of theorem 3.1 gives the estimates:

$$
\left(\frac{u\left(N \epsilon^{p}+\mu, t \epsilon^{q}+\chi\right)}{v\left(N \epsilon^{p}+\mu, t \epsilon^{q}+\chi\right)}\right)^{ \pm 1} \leq(2 M)^{8 P_{A(N, t)}(c)}[u: v]_{(L+1) \epsilon}^{c^{A(N, t)}}
$$

for any $0 \leq \mu \leq \epsilon^{p}$ and $0 \leq \chi \leq \epsilon^{q}$. Then we have the estimates:

$$
\begin{aligned}
A(N, t)= & (t-d-1) k+N-l+n-1 \\
& \leq \epsilon^{-q} k\left(t \epsilon^{q}+\chi\right)-d k+\epsilon^{-p}\left(N \epsilon^{p}+\mu\right)-l+n \\
& \leq \epsilon^{-q} k\left(t \epsilon^{q}+\chi\right)+\epsilon^{-p}\left(N \epsilon^{p}+\mu\right)+n \\
& \leq \epsilon^{-\alpha}\left[k\left(t \epsilon^{q}+\chi\right)+\left(N \epsilon^{p}+\mu\right)\right]+n
\end{aligned}
$$

where $\alpha=\max (p, q)$. Then:

$$
\begin{aligned}
& (2 M)^{8 P_{A(N, t)}(c)}[u: v]_{(L+1) \epsilon}^{c^{A(N, t)}} \\
& \leq(2 M)^{8 \frac{\epsilon^{\epsilon^{-D}\left[k\left(t \epsilon^{q}+\chi\right)+\left(N \epsilon^{p}+\mu\right)\right]+1}-1}{c-1}}[u: v]_{(L+1) \epsilon}^{c^{\epsilon^{-D}\left[k\left(t \epsilon^{q}+\chi\right)+\left(N \epsilon^{p}+\mu\right)\right]+n}} .
\end{aligned}
$$

Now combing with these estimates, one obtains the desired estimates:

$$
\left(\frac{u(x, s)}{v(x, s)}\right)^{ \pm 1} \leq(2 M)^{8^{\frac{\epsilon^{-D}(x+k s)+1}{c-1}}}[u: v]_{(L+1) \epsilon}^{c^{\epsilon^{-D}(x+k s)+n}} .
$$

This completes the proof. 
Example: Let $b>a \geq 1$ be positive integers, and consider linear PDEs $\mathbf{F}\left(v_{x}, v_{s}\right)=a v_{x}+b v_{s}=0$. For increasing and relative elementary functions $f$ with its leading term $\mathbf{F}$, let us consider the discrete dynamics:

$$
z_{N+1}^{t+1}=f\left(z_{N}^{t}, z_{N+1}^{t}\right)=\frac{1}{b}\left(a z_{N}^{t}+(b-a) z_{N+1}^{t}\right) .
$$

Let $v:(0, \infty) \times[0, \infty) \rightarrow(0, \infty)$ be $C^{2}$ functions, and take the Taylor expansions up to order 2 . We choose the scaling parameters by $N=\frac{x}{\epsilon}, t=\frac{s}{\epsilon}$ and $z_{N}^{t}=v(x, s)$, and insert the Taylor expansions:

$$
\begin{aligned}
& v(x+\epsilon, s+\epsilon)-f(v(x, s), v(x+\epsilon, s)) \\
& =\frac{\epsilon}{b}\left(a v_{x}+b v_{s}\right)+\frac{\epsilon^{2}}{2}\left(\left(v_{2 x}+v_{2 s}+2 v_{x s}\right)\left(\eta_{1}\right)-\frac{b-a}{b} v_{2 x}\left(\eta_{2}\right)\right) .
\end{aligned}
$$

$f$ correspond to $V_{N+1}^{t+1}=\max \left(V_{N}^{t}, \ldots, V_{N}^{t}, V_{N+1}^{t}, \ldots, V_{N+1}^{t}\right)-\max (0, \ldots, 0)$, where their terms iterate $a, b-a$ and $b$ times respecively. Clearly this shows that $f$ are all tropically equivalent indpendently of $b>a \geq 1$.

By lemma 2.2, the Lipschitz constants $c_{f}=1$ are all equal to one. For the numbers of the components, $M_{f}=b^{2}$ hold. $D=1, L=0, n=2$ and $k=1$. For any positive integers $a, b, a^{\prime}, b^{\prime}$, let us take two solutions $u(x, s)$ and $v(x, s)$ satisfying the equations $a u_{x}+b u_{s}=0$ and $a^{\prime} v_{x}+b^{\prime} v_{s}=0$ respecively. One may assume $b \geq b^{\prime}$. Suppose both are $\epsilon_{0}$ controlled bounded by $C$. Then by corollary 3.4 , for any $0<\epsilon \leq \min \left(\frac{1}{2 C}, \epsilon_{0}\right)$, the exponential estimates must hold:

$$
\left(\frac{u(x, s)}{v(x, s)}\right)^{ \pm 1} \leq\left(2 b^{2}\right)^{8\left(\epsilon^{-1}(x+s)+1\right)}[u: v]_{\epsilon} .
$$

Below we apply the general procedure of the previous sections to non linear partial differential equations. We treat two PDEs, where one is the quasi linear equations of order 1 , and the other is diffusion equations. Given PDE, then our procedure is to find 'good' relative elementary functions $f$. We have to require them to be increasing. Any elementary polynimials are increasing. One of applicable form of $f$ is:

$$
f\left(z_{1}, z_{2}, \ldots\right)=\frac{z_{1}\left(\alpha+P\left(z_{1}, z_{2}, \ldots\right)\right)}{1+z_{1}}+Q\left(z_{1}, \ldots\right)
$$

where both $P$ and $Q$ are elementary polynomials and $0 \leq \alpha \leq 1$.

One may weaken the required properties, if both the range and the domain for disctete dynamics are within the regions of monotone increasing for these functions. 


\section{Applications}

4.A Quasi linear equations: Here we introduce a cancelation method of non linear terms and use it to compare solutions between the following equations. Let us consider the equations of the form:

$$
v_{s}+\epsilon v v_{x}-\frac{1}{2} v^{2}=0, \quad 2 u_{s}+\epsilon u\left(u_{s}+u_{x}\right)=0
$$

where $\epsilon>0$ are small constants. These two types of the equations differ from each other, in that for the right hand side, each monomial contains differentials of $u$, and so in particular any constants are solutions. Notice that $v(x, s)=\frac{c}{1-0.5 c s}$ are degenerate solutions on $(0, \infty) \times\left[0, \frac{2}{c}\right)$ for $c>0$.

We choose the second variation:

$$
\|u\|_{2}(x, s)=\sup _{\xi-(x-\epsilon, s-\epsilon) \in D(\epsilon, 1,1)}\left\{\left|\frac{\partial^{2} u}{\partial x^{2}}\right|(\xi),\left|\frac{\partial^{2} u}{\partial s^{2}}\right|(\xi),\left|\frac{\partial^{2} u}{\partial x \partial s}\right|(\xi)\right\}
$$

and put the variation constant:

$$
V(u) \equiv \sup _{(x, s) \in(0, \infty) \times\left[0, T_{0}\right)} \frac{\|u\|_{2}(x, s)}{u(x, s)} .
$$

Let us fix any positive constant $V_{0}>0$.

Theorem 4.1. For any $0<\epsilon \leq 0.1 V_{0}^{-1}$, let $v, u:(0, \infty) \times\left[0, T_{0}\right) \rightarrow(0, \infty)$ be $C^{2}$ solutions to the quasi linear equations:

$$
v_{s}+\epsilon v v_{x}-\frac{1}{2} v^{2}=0, \quad 2 u_{s}+\epsilon u\left(u_{s}+u_{x}\right)=0 .
$$

Suppose their variation constants $V(u), V(v)$ are bounded by $V_{0}$. Then they satisfy the asymptotic estimates for all $(x, s) \in(0, \infty) \times\left[0, T_{0}\right)$ :

$$
\left(\frac{u(x, s)}{v(x, s)}\right)^{ \pm 1} \leq 40^{2^{\epsilon^{-1}(x+2 s)+4}}\left([u: v]_{2 \epsilon}\right)^{2^{\epsilon^{-1}(x+2 s)+3}} .
$$

In particular when $u(x, s) \equiv R>0$ is constant, then the estimates hold:

$$
\begin{aligned}
R(40)^{-2^{\epsilon^{-1}(x+2 s)+4}}\left([v: R]_{2 \epsilon}\right)^{-2^{\epsilon^{-1}(x+2 s)+3}} & \\
& \leq v(x, s) \leq R(40)^{2^{\epsilon^{-1}(x+2 s)+4}}\left([v: R]_{2 \epsilon}\right)^{2^{\epsilon^{-1}(x+2 s)+3}} .
\end{aligned}
$$


4.A.2 Induction of the equations: Let us consider the dynamics:

$$
z_{N+1}^{t+1}=f\left(z_{N}^{t}, z_{N+2}^{t}, z_{N-1}^{t+1}\right) \equiv \frac{z_{N+2}^{t}}{2}+\frac{z_{N}^{t}\left(1+2 z_{N-1}^{t+1}\right)}{2\left(1+z_{N}^{t}\right)} .
$$

$f$ is an increasing function. The corresponding $(\max ,+)$-function is given by $\max \left(V_{N+2}^{t}, V_{N+2}^{t}+V_{N}^{t}, V_{N}^{t}, V_{N}^{t}+V_{N-1}^{t+1}, V_{N}^{t}+V_{N-1}^{t+1}\right)-\max \left(0,0, V_{N}^{t}, V_{N}^{t}\right)$. The number of the components is $M=5 \times 4=20$, and its Lipschitz constant is equal to 2 .

We choose the scaling parameters by:

$$
\epsilon v(x, s)=z_{N}^{t}, \quad N=\frac{x}{\epsilon}, \quad t=\frac{s}{\epsilon}
$$

where we take a small $\epsilon>0$ so that the estimate $\epsilon \leq 0.1 V_{0}^{-1}$ holds.

Let $v:(0, \infty) \times\left[0, T_{0}\right) \rightarrow(0, \infty)$ be a $C^{2}$ function, and take the Taylor expansions up to order 2 :

$$
\begin{aligned}
v(x+i \epsilon, s+j \epsilon) \\
\quad=v+i \epsilon v_{x}+j \epsilon v_{s}+\epsilon^{2}\left(\frac{i^{2}}{2} v_{2 x}+\frac{j^{2}}{2} v_{2 s}+i j v_{x s}\right)\left(\xi_{i j}\right) \\
\quad \equiv v+i \epsilon v_{x}+j \epsilon v_{s}+\epsilon^{2} D^{2} v\left(\xi_{i j}\right) .
\end{aligned}
$$

Let us insert the formal Taylor expansions:

$$
\begin{aligned}
& \epsilon v(x+\epsilon, s+\epsilon)-f(\epsilon v(x, s), \epsilon v(x+2 \epsilon, s), \epsilon v(x-\epsilon, s+\epsilon)) \\
& \left.=\frac{1}{2(1+\epsilon v)}\left[\epsilon^{2}\left(2 v_{s}+2 \epsilon v v_{x}-v^{2}\right)-2(\epsilon v) \epsilon^{2} D^{2} v\left(\xi_{-11}\right)\right\}\right] \\
& \quad+\epsilon^{2}\left(D^{2} v\left(\xi_{11}\right)-\frac{1}{2} D^{2} v\left(\xi_{20}\right)\right)
\end{aligned}
$$

where the leading term is given by:

$$
\mathbf{F}=\epsilon^{2} \frac{2 v_{s}+2 \epsilon v v_{x}-v^{2}}{2(1+\epsilon v)}
$$

The error term is admissible, and let us calculate the error constant $C_{\mathbf{F}^{1}}$. Notice the estimates $\left|D^{2} v\left(\xi_{i j}\right)\right| \leq\left(\frac{i^{2}+j^{2}}{2}+|i j|\right)\|v\|_{2}(x, s)$, where $\|v\|_{2}(x, s)$ is the second variation. Then the error term satisfies the estimates:

$\left\|\mathbf{F}^{1}\right\|_{\epsilon}(x, s) \leq \frac{2(\epsilon v) \epsilon^{2}\left|D^{2} v\left(\xi_{-11}\right)\right|}{2(1+\epsilon v)}+\epsilon^{2}\left(\left|D^{2} v\left(\xi_{11}\right)\right|+\left|\frac{1}{2} D^{2} v\left(\xi_{20}\right)\right|\right) \leq 5 \epsilon^{2}\|v\|_{2}(x, s)$. 
In particular the error constant is given by:

$$
C_{\mathbf{F}^{1}}=5 .
$$

4.A.3 Deformation and cancelation: Let us introduce a cancelation method below. Let us consider the discrete dynamics:

$$
w_{N+1}^{t+1}=g\left(w_{N}^{t}, w_{N+2}^{t}, w_{N-1}^{t+1}\right) \equiv \frac{w_{N+2}^{t}}{2}+\frac{w_{N}^{t}+w_{N}^{t} w_{N-1}^{t+1}}{2\left(1+w_{N}^{t}\right)} .
$$

$g$ is also an increasing function and is tropically equivalent to $f$. The number of the components is 16 , and the corresponding $(\max ,+)$-function has its Lipschitz constant 2.

Let $u:(0, \infty) \times\left[0, T_{0}\right) \rightarrow(0, \infty)$ be a $C^{2}$ function, and choose the same scaling parameters, $\epsilon u(x, s)=z_{N}^{t}, N=\frac{x}{\epsilon}$ and $t=\frac{s}{\epsilon}$. Then let us insert the Taylor expansions of $u$ up to order 2 into the difference as before. Then the direct calculation shows that unlike to the previous case, $u^{2}$ term is eliminated, and the result is given by:

$$
\begin{aligned}
& \epsilon u(x+\epsilon, s+\epsilon)-g(\epsilon u(x, s), \epsilon u(x+2 \epsilon, s), \epsilon u(x-\epsilon, s+\epsilon)) \\
& =\frac{1}{2(1+\epsilon u)}\left[\epsilon^{2}\left(2 u_{s}+\epsilon u u_{s}+\epsilon u u_{x}\right)-\epsilon u \epsilon^{2} D^{2} u\left(\eta_{-11}\right)\right] \\
& \quad+\epsilon^{2}\left(D^{2} u\left(\eta_{11}\right)-\frac{1}{2} D^{2} u\left(\eta_{20}\right)\right)
\end{aligned}
$$

where the leading term is given by:

$$
\mathbf{G}=\epsilon^{2} \frac{2 u_{s}+\epsilon u u_{s}+\epsilon u u_{x}}{2(1+\epsilon u)} .
$$

In this deformation also, the error term is admissible, and satisfies the estimates:

$$
\left\|\mathbf{G}^{1}\right\|_{\epsilon}(x, s) \leq \frac{\epsilon u \epsilon^{2}\left|D^{2} u\left(\eta_{-11}\right)\right|}{2(1+\epsilon u)}+\epsilon^{2}\left(\left|D^{2} u\left(\eta_{11}\right)\right|+\left|\frac{1}{2} D^{2} u\left(\eta_{20}\right)\right|\right) \leq 4 \epsilon^{2}\|u\|_{2}(x, s) .
$$

So the error constant is give by $C_{\mathbf{G}^{1}}=4$.

Proof of theorem 4.1: Let $u, v:(0, \infty) \times\left[0, T_{0}\right) \rightarrow(0, \infty)$ be $C^{2}$ functions which satisfy the equations $v_{s}+\epsilon v v_{x}-\frac{1}{2} v^{2}=0$ and $2 u_{s}+\epsilon u\left(u_{s}+u_{x}\right)=0$. 
Suppose they have bounded variation constants $V(u), V(v) \leq V_{0}$. Then by applying corollary 3.4 and lemma 3.2 , one obtains the asymptotic estimates:

$$
\left(\frac{u(x, s)}{v(x, s)}\right)^{ \pm 1} \leq(2 M)^{8 \frac{\epsilon^{\epsilon^{-D}(x+k s)+1}-1}{c-1}}\left([u: v]_{(L+1) \epsilon}\right)^{c^{\epsilon^{-D}(x+k s)+n}}
$$

for any $0<\epsilon \leq\left(2 C V_{0}\right)^{-1}$, where in this case $D=\max (p, q)=1, C=5$, $L=1, M=20, c=2, k=2$ and $n=3$. Thus for any $0<\epsilon \leq 0.1 V_{0}^{-1}$, the estimates:

$$
\left(\frac{u(x, s)}{v(x, s)}\right)^{ \pm 1} \leq(40)^{2^{-1}(x+2 s)+4}\left([u: v]_{2 \epsilon}\right)^{2^{\epsilon^{-1}(x+2 s)+3}}
$$

hold. This completes the proof.

4.B Diffusion equations: Here we introduce a linear deformation of elementary functions, and use it to compare between solutions to different diffusion equations.

Let $F$ be a relative elementary and increasing function of one variable. Here we consider the diffusion equations of the type:

$$
u_{s}=u_{2 x}+F(u) \text {. }
$$

We take the third variation:

$$
\|u\|_{3}(x, s)=\sup _{\xi-\left(x-\epsilon^{2}, s-\epsilon\right) \in D(\epsilon, 2,1)}\left\{\left|\frac{\partial^{3} u}{\partial x^{3}}\right|(\xi),\left|\frac{\partial^{3} u}{\partial s^{3}}\right|(\xi),\left|\frac{\partial^{3} u}{\partial x^{2} \partial s}\right|(\xi),\left|\frac{\partial^{3} u}{\partial x \partial s^{2}}\right|(\xi)\right\}
$$

and put the variation constant:

$$
V(u) \equiv \sup _{(x, s) \in(0, \infty) \times\left[0, T_{0}\right)} \frac{\|u\|_{3}(x, s)}{u(x, s)} .
$$

4.B.2 Linear deformations: Let $F$ be relative elementary and increasing, or zero. We consider the discrete dynamics of the form:

$$
z_{N+1}^{t+1}=f\left(z_{N-1}^{t-4}, z_{N-4}^{t-1}, z_{N}^{t}, z_{N+4}^{t}\right)=\alpha z_{N-4}^{t-1}+\beta z_{N-1}^{t-4}+\gamma z_{N}^{t}+\delta z_{N+4}^{t}+F\left(z_{N}^{t}\right)
$$

where $\alpha, \beta, \gamma, \delta>0$ are all positive rational numbers.

We choose the scaling parameters by

$$
z_{N}^{t}=\epsilon^{l} u(x, s), \quad N=\frac{x}{\epsilon^{m}}, \quad t=\frac{s}{\epsilon^{2 m}}, \quad(l \geq 0, m \geq 1) .
$$


For a $C^{3}$ function $u:(0, \infty) \times\left[0, T_{0}\right) \rightarrow(0, \infty)$, let us take the Taylor expansions as before:

$$
\begin{aligned}
u\left(x+i \epsilon^{m}, s\right. & \left.+j \epsilon^{2 m}\right)=u+i \epsilon^{m} u_{x}+j \epsilon^{2 m} u_{s} \\
& +i^{2} \frac{\epsilon^{2 m}}{2} u_{2 x}+j^{2} \frac{\epsilon^{4 m}}{2} u_{2 s}+i j \epsilon^{3 m} u_{x s}+\epsilon^{3 m} D_{\epsilon}^{3} u\left(\xi_{i j}\right) .
\end{aligned}
$$

Firstly we consider the differences:

$$
z_{N+1}^{t+1}-\left(\frac{p}{4} z_{N-4}^{t-1}+\frac{1-p}{4} z_{N-1}^{t-4}\right)
$$

for $0<p<1$. It is immediate to see that this does not contain $u_{x s}$ term. Let us determine $p \in \mathbb{Q}$ so that it also contains no $u_{2 s}$ term. In fact for $p=\frac{4}{5}$, the difference is:

$$
\frac{3}{4} \epsilon^{l} u+\frac{7}{5} \epsilon^{l+2 m} u_{s}+\frac{37}{20} \epsilon^{l+m} u_{x}-\frac{9}{8} \epsilon^{l+2 m} u_{2 x}+\epsilon^{l+3 m} \text { Higher terms }
$$

where Higher terms consiste of linear combinations of three derivatives.

Next we eliminate $u_{x}$ term by adding $\delta z_{N+4}^{t}$ for $\delta=\frac{37}{80}$, and then finally we eliminate $u$ terms by adding $\gamma z_{N}^{t}$ for $\gamma=\frac{23}{80}$ :

$$
\begin{aligned}
z_{N+1}^{t+1}- & \left(\frac{1}{5} z_{N-4}^{t-1}+\frac{1}{20} z_{N-1}^{t-4}+\frac{37}{80} z_{N+4}^{t}+\frac{23}{80} z_{N}^{t}\right) \\
& =\epsilon^{l+2 m}\left(\frac{7}{5} u_{s}-\frac{193}{40} u_{2 x}\right)+\epsilon^{l+3 m} \text { Higher terms }
\end{aligned}
$$

Next if we choose constants as below, then one induces the following:

$$
\begin{aligned}
& w_{N+1}^{t+1}-g\left(w_{N-1}^{t-4}, w_{N-4}^{t-1}, w_{N}^{t}, w_{N+4}^{t}\right) \\
& \equiv w_{N+1}^{t+1}-\left(\frac{1}{24} w_{N-4}^{t-1}+\frac{5}{24} w_{N-1}^{t-4}+\frac{1}{128} w_{N+4}^{t}+\frac{95}{128} w_{N}^{t}\right) \\
& =\epsilon^{l+m}\left(\frac{15}{8} \epsilon^{m} v_{s}+\frac{43}{32} v_{x}-\frac{19}{16} \epsilon^{3 m} v_{2 s}\right)+\epsilon^{l+3 m} \text { Higher terms } .
\end{aligned}
$$

$g$ and $f$ above are mutually tropically equivalent. If one exchanges the role of variables and regards $x$ as the time parameter, then the first term of the right hand side equation gives the advection-diffusion equation.

Proposition 4.2. Let us fix $V_{0}>0$ and choose any $0<\epsilon \leq\left(200 V_{0}\right)^{-1}$. Let $u, v:(0, \infty) \times[0, \infty) \rightarrow(0, \infty)$ be $C^{3}$ solutions to the linear equations:

$$
\frac{7}{5} u_{s}-\frac{193}{40} u_{2 x}=0, \quad \frac{15}{8} \epsilon v_{s}+\frac{43}{32} v_{x}-\frac{19}{16} \epsilon^{3} v_{2 s}=0 .
$$


Suppose their variation constants satisfy the bounds $V(u), V(v) \leq V_{0}$. Then they satisfy the exponential asymptotic estimates for all $(x, s) \in(0, \infty) \times$ $[0, \infty)$ :

$$
\left(\frac{u(x, s)}{v(x, s)}\right)^{ \pm 1} \leq 10^{48\left(\epsilon^{-2}(x+4 s)+1\right)}[u: v]_{5 \epsilon} .
$$

Proof: Let us consider two linear functions:

$$
\begin{aligned}
& f\left(z_{N-1}^{t-4}, z_{N-4}^{t-1}, z_{N}^{t}, z_{N+4}^{t}\right)=\frac{1}{5} z_{N-4}^{t-1}+\frac{1}{20} z_{N-1}^{t-4}+\frac{37}{80} z_{N+4}^{t}+\frac{23}{80} z_{N}^{t}, \\
& g\left(w_{N-1}^{t-4}, w_{N-4}^{t-1}, w_{N}^{t}, w_{N+4}^{t}\right)=\frac{1}{24} w_{N-4}^{t-1}+\frac{5}{24} w_{N-1}^{t-4}+\frac{1}{128} w_{N+4}^{t}+\frac{95}{128} w_{N}^{t}
\end{aligned}
$$

Let us choose $m=1$. Then the estimates in corollary 3.4 and lemma 3.2 give the following for $0<\epsilon \leq\left(2 C V_{0}\right)^{-1}$ :

$$
\left(\frac{u(x, s)}{v(x, s)}\right)^{ \pm 1} \leq(2 M)^{8 \frac{c^{\epsilon^{-D}(x+k s)+1}-1}{c-1}}\left([u: v]_{(L+1) \epsilon}\right)^{c^{\epsilon^{-D}(x+k s)+n}} .
$$

For the corresponding $(\max ,+)$-functions, their Lipschitz constants are both $c=1$, and the numbers of the components are bounded roughly by $M \leq \frac{10^{6}}{2}$. For both cases, the error terms are the Higher terms above, consisted by the linear combinations of the three derivatives. So the error constants are roughly bounded by $\frac{1}{6} \times 8 \times 4^{3} \leq 10^{2} . k=4, D=\max (p, q)=2$ and $L=\max (l, d)=4$. So in this case for any $0<\epsilon \leq\left(200 V_{0}\right)^{-1}$, the estimates:

$$
\left(\frac{u(x, s)}{v(x, s)}\right)^{ \pm 1} \leq 10^{48\left(\epsilon^{-2}(x+4 s)+1\right)}[u: v]_{5 \epsilon}
$$

hold. This completes the proof.

4.B.3 Non deforming: Let us consider the non linear diffusion equations:

$$
u_{s}=u_{2 x}+u^{a}, \quad 1<a \in \mathbb{Q} .
$$

In order to estimate its asymptotics, one considers $v:(0, \infty) \times\left[0, T_{0}\right) \rightarrow$ $(0, \infty)$, which is a $C^{3}$ solution to the equation $v_{s}=v^{a}$. For the initial value $c>0$, this is easily solved as:

$$
v(s)=\frac{c}{\left(1-c^{a-1}(a-1) s\right)^{(a-1)^{-1}}} .
$$


The blowing up time is $S_{0}=\frac{1}{c^{a-1}(a-1)}$. Its three derivative is given by $\frac{d^{3} v(s)}{d s^{3}}=$ $\frac{c^{1+3 \alpha^{-1}}(\alpha+1)(\alpha+2)}{\alpha^{2}\left(1-c^{\alpha^{-1}} \alpha^{-1} s\right)^{\alpha+3}}$, where $\alpha=(a-1)^{-1}$. Thus for $0 \leq s \leq s_{0}<S_{0}$, the variation constant $V$ is bounded by:

$$
V\left(s_{0}\right)=\frac{c^{3 \alpha^{-1}}(\alpha+1)(\alpha+2)}{\alpha^{2}\left(1-c^{\alpha^{-1}} \alpha^{-1} s_{0}\right)^{3}} .
$$

Conversely for any $V(0) \leq V_{0}<\infty$, there are unique $s_{0}<S_{0}$ so that the equality $V_{0}=V\left(s_{0}\right)$ holds.

Theorem 4.3. Let us fix any $V_{0}=V\left(s_{0}\right)$. For any $1<a \in \mathbb{Q}$ and $T_{0} \leq s_{0}$, let $u:(0, \infty) \times\left[0, T_{0}\right) \rightarrow(0, \infty)$ be $C^{3}$ solutions to the diffusion equations:

$$
u_{s}-u_{2 x}=u^{a} .
$$

Suppose their variation constants $V(u)$ are bounded by $V_{0}$. Then for any $0<\epsilon \leq\left(200 V_{0}\right)^{-1}$, u satisfies the asymptotic estimates:

$$
\left(\frac{u(x, s)}{v(s)}\right)^{ \pm 1} \leq 10^{40 \frac{a^{\epsilon^{-2}(2 x+4 s)+1}-1}{a-1}}\left([u: v]_{5 \epsilon}\right)^{a^{\epsilon^{-2}(2 x+4 s)+4}} .
$$

Proof: In 4.B.2, let us choose the rescaling parameters $m=1$ and $l \in \mathbb{Q}$ so that the equality $l+2 m=l a$ holds. In order to induce the above non linear diffusion equations from discrete dynamics, we add non linear term.

Let $\tilde{u}:(0, \infty) \times\left[0, T_{0}\right) \rightarrow(0, \infty)$ be a $C^{3}$ function, and consider the discrete dynamics:

$$
\begin{aligned}
& z_{N+1}^{t+1}-f\left(z_{N-1}^{t-4}, z_{N-4}^{t-1}, z_{N}^{t}, z_{N+4}^{t}\right) \\
& =z_{N+1}^{t+1}-\left(\frac{1}{5} z_{N-4}^{t-1}+\frac{1}{20} z_{N-1}^{t-4}+\frac{37}{80} z_{N+4}^{t}+\frac{23}{80} z_{N}^{t}+\frac{7}{5}\left(z_{N}^{t}\right)^{a}\right) \\
& =\epsilon^{l a}\left(\frac{7}{5} \tilde{u}_{s}-\frac{193}{40} \tilde{u}_{2 x}-\frac{7}{5} \tilde{u}^{a}\right)+\epsilon^{l+3} \text { Higher terms. }
\end{aligned}
$$

For the corresponding (max, + )-function to $f$, the Lipschitz constant is $a>1$, and the number of the components are bounded roughly by $\frac{1}{2} 10^{5}$. The error constant is again roughly bounded by $10^{2}, k=4, D=2$ and $L=4$.

Suppose $\tilde{u}$ satisfies the equation $\frac{7}{5} \tilde{u}_{s}-\frac{193}{40} \tilde{u}_{2 x}-\frac{7}{5} \tilde{u}^{a}=0$ which admits bounded variation constants $V(\tilde{u}) \leq \tilde{V}$. Then by corollary 3.4 and lemma 3.2 , one finds the asymptotic estimates for $0<\epsilon \leq(200 \tilde{V})^{-1}$ :

$$
\left(\frac{\tilde{u}(x, s)}{v(s)}\right)^{ \pm 1} \leq 10^{40 \frac{a^{\epsilon^{-2}(x+4 s)+1}-1}{a-1}}\left([\tilde{u}: v]_{5 \epsilon}\right)^{a^{\epsilon^{-2}(x+4 s)+4}} .
$$


Let us change the variable $x$ as $\tilde{u}(x, s)=u(p x, s)$, where $\frac{1}{2} \leq p=$ $\sqrt{\frac{40}{193} \times \frac{7}{5}} \leq 1$, and put the variation constants of $u$ by $V(u)$. Notice that if $u_{s}-u_{2 x}-u^{a}=0$ holds, then $\tilde{u}$ satisfies the equation $\frac{7}{5} \tilde{u}_{s}-\frac{193}{40} \tilde{u}_{2 x}-\frac{7}{5} \tilde{u}^{a}=0$. Their variation constants satisfy the estimates $V(\tilde{u}) \leq V(u)$. Thus the asymptotic estimates hold for $0<\epsilon \leq\left(200 V_{0}\right)^{-1}$ :

$$
\left(\frac{u(x, s)}{v(s)}\right)^{ \pm 1} \leq 10^{40 \frac{a^{\epsilon^{-2}(2 x+4 s)+1}-1}{a-1}}\left([u: v]_{5 \epsilon}\right)^{a^{\epsilon^{-2}(2 x+4 s)+4}} .
$$

This completes the proof.

Remark: Notice that the third derivative rates for $v:\left[0, s_{0}\right] \rightarrow(0, \infty)$ are given by:

$$
K\left(s_{0}\right)=\frac{c^{3 \alpha^{-1}}(\alpha+1)(\alpha+2)}{\alpha^{2}\left(1-c^{\alpha^{-1}} \alpha^{-1} s_{0}\right)^{\alpha+3}} .
$$

4.B.4 Inhomogeneous non linear equations: The above method does not work for diffusion equations with inhomogeneous non linear terms. In order to treat such cases, we use tropical deformations for relative elementary functions. Here we treat diffusion equations of the form:

$$
u_{s}-u_{2 x}-u^{a}-\delta u^{b}=0, \quad(1<a<b, 0<\delta<<1) .
$$

Here we cover the equations of the types:

$$
(a, b)=\left(1+\alpha^{-1}, 1+2 \alpha^{-1}\right), \quad 0.5 \leq \alpha \leq 1 .
$$

Let $\mu=\frac{p}{q} \in \mathbb{Q}_{>0}$ be positive rational numbers, where $p, q \in \mathbb{N}$ are relatively prime numbers. We put $c_{\mu} \equiv p q \in \mathbb{Z}_{>0}$ and call them as the number of the components for $\mu$.

For $\alpha \in \mathbb{Q}$ and $c>0$, let us put:

$$
\begin{aligned}
& V\left(s_{0}\right)=\frac{c^{3 \alpha^{-1}} 5^{3}(\alpha+1)(\alpha+2)}{6^{3} \alpha^{2}\left(1-c^{\prime} s_{0}\right)^{3}}, \quad c^{\prime}=\frac{5 c^{\alpha^{-1}}}{6 \alpha}, \\
& (a, b)=\left(1+\alpha^{-1}, 1+2 \alpha^{-1}\right), \quad \delta=\mu \epsilon^{2}, \quad \mu=\frac{\alpha+1}{9 \alpha}
\end{aligned}
$$

for $0 \leq s_{0}<\left(c^{\prime}\right)^{-1}$. Let us compare $u$ with the function:

$$
v(s)=\frac{c}{\left(1-c^{\prime} s\right)^{\alpha}} .
$$

Let us fix any $V_{0}=V\left(s_{0}\right) \geq V(0)$. 
Theorem 4.4. For any $0<T_{0} \leq s_{0}$ and any $0<\epsilon \leq\left(200 V_{0}\right)^{-1}$, let $u:(0, \infty) \times\left[0, T_{0}\right) \rightarrow(0, \infty)$ be $C^{3}$ solutions to the the diffusion equations:

$$
u_{s}-u_{2 x}=u^{a}+\delta u^{b} .
$$

Suppose their variation constants $V(u)$ are bounded by $V_{0}$. Then $u$ satisfies the asymptotic estimates:

$$
\left(\frac{u(x, s)}{v(s)}\right)^{ \pm 1} \leq\left(2 M_{\mu}\right)^{8^{\frac{\epsilon^{-2}(2 x+4 s)+1}{b-1}}}\left([u: v]_{5 \epsilon}\right)^{b^{\epsilon^{-2}(2 x+4 s)+4}}
$$

where $M_{\mu}=\max \left(2 \times 10^{3} c_{\mu}^{2}, 3 \times 10^{4}\right)$.

Proof: Firstly let us consider the tropical deformation:

$$
\begin{aligned}
& w_{N+1}^{t+1}-g\left(w_{N-1}^{t-4}, w_{N-4}^{t-1}, w_{N}^{t}, w_{N+4}^{t}\right)=w_{N+1}^{t+1}- \\
& \left(\frac{1}{25}\left(w_{N-4}^{t-1}+w_{N-1}^{t-4}\right)+\frac{1}{25} w_{N+4}^{t}+\frac{22}{25} w_{N}^{t}+\left(w_{N}^{t}\right)^{a}+\mu\left(w_{N}^{t}\right)^{b}\right) \\
& =\epsilon^{l}\left(\epsilon^{2 m}\left(\frac{6}{5} v_{s}+\frac{4}{25} \epsilon^{2 m} v_{2 s}\right)+\frac{26}{25} \epsilon^{m} v_{x}+\frac{17}{25} \epsilon^{3 m} v_{x s}-\frac{4}{25} \epsilon^{2 m} v_{2 x}\right) \\
& -\left(\left(w_{N}^{t}\right)^{a}+\mu\left(w_{N}^{t}\right)^{b}\right)+\epsilon^{l+3 m} \text { Higher terms } \\
& =\left[\epsilon^{l+2 m}\left(\frac{6}{5} v_{s}+\frac{4}{25} \epsilon^{2 m} v_{2 s}\right)-\epsilon^{a l} v^{a}-\mu \epsilon^{b l} v^{b}\right] \\
& +\epsilon^{l}\left(\frac{26}{25} \epsilon^{m} v_{x}+\frac{17}{25} \epsilon^{3 m} v_{x s}-\frac{4}{25} \epsilon^{2 m} v_{2 x}\right)+\epsilon^{l+3 m} \text { Higher terms. }
\end{aligned}
$$

Since $\mu \leq \frac{1}{3}$ hold, the number of the components for $g$ is bounded by $75 \times 25 c_{\mu}^{2} \leq 2 \times 10^{3} c_{\mu}^{2}$. The corresponding Lipschitz constant is $b . \quad D=$ $\max (p, q)=2 m$ and $L=4$. The error constants are bounded by $\frac{3 \times 8}{25 \times 6} \times 4^{3} \leq$ 11.

Sublemma 4.5. For $(a, b)=\left(1+\alpha^{-1}, 1+2 \alpha^{-1}\right), 0.5 \leq \alpha \leq 1$, one can choose $l \in \mathbb{Q}$ and $m=1$ so that both the equalities $l+2 m=a l$ and $(b-a) l=2 m$ hold.

Proof: By the condition, $m=\frac{b-a}{2} l$ must hold. By inserting into the first condition, one obtains the equality $1+(b-a)=a$, which certainly hold for the above pairs $(a, b)$. This completes the proof.

If one chooses $l \in \mathbb{Q}$ and $m=1$ as above, then the equality holds:

$$
\epsilon^{l+2}\left(\frac{6}{5} v_{s}+\frac{4}{25} \epsilon^{2} v_{2 s}\right)-\epsilon^{a l} v^{a}-\mu \epsilon^{b l} v^{b}=\epsilon^{l+2}\left(\frac{6}{5} v_{s}+\frac{4}{25} \epsilon^{2} v_{2 s}-v^{a}-\mu \epsilon^{2} v^{b}\right) .
$$


Sublemma 4.6. Moreover let us put $\mu=\frac{\alpha+1}{9 \alpha}$. Then for any $c>0, v(s)=$ $\frac{c}{\left(1-c^{\prime} s\right)^{\alpha}}\left(c^{\prime}=\frac{5 c^{\alpha^{-1}}}{6 \alpha}\right)$ satisfy the equations:

$$
\frac{6}{5} v_{s}+\frac{4}{25} \epsilon^{2} v_{2 s}-v^{a}-\mu \epsilon^{2} v^{b}=0 .
$$

This can be checked by direct calculations. Notice it satisfies the equation $\epsilon^{l+2 m}\left(\frac{6}{5} v_{s}+\frac{4}{25} \epsilon^{2 m} v_{2 s}\right)-\epsilon^{a l} v^{a}-\mu \epsilon^{b l} v^{b}+\epsilon^{l}\left(a_{1} \epsilon^{m} v_{x}+a_{2} \epsilon^{3 m} v_{x s}-a_{3} \epsilon^{2 m} v_{2 x}\right)=0$.

Proof of theorem: Three derivative of $v$ is given by $\frac{d^{3} v(s)}{d s^{3}}=\frac{c^{1+3 \alpha^{-1}} 5^{3}(\alpha+1)(\alpha+2)}{6^{3} \alpha^{2}\left(1-c^{\prime} s\right)^{\alpha+3}}$. Thus for $0 \leq s \leq s_{0}<S_{0}=\left(c^{\prime}\right)^{-1}$, the variation constants are bounded by:

$$
V\left(s_{0}\right)=\frac{c^{3 \alpha^{-1}} 5^{3}(\alpha+1)(\alpha+2)}{6^{3} \alpha^{2}\left(1-c^{\prime} s_{0}\right)^{3}} .
$$

As before for any $V(0) \leq V_{0}<\infty$, there are unique $s_{0}<S_{0}$ so that the equality $V_{0}=V\left(s_{0}\right)$ holds.

For $0<T_{0} \leq s_{0}$, let $\tilde{u}:(0, \infty) \times\left[0, T_{0}\right) \rightarrow(0, \infty)$ be $C^{3}$ functions, and consider the discrete dynamics:

$$
\begin{aligned}
& z_{N+1}^{t+1}-f\left(z_{N-1}^{t-4}, z_{N-4}^{t-1}, z_{N}^{t}, z_{N+4}^{t}\right) \\
& =z_{N+1}^{t+1}-\left(\frac{1}{5} z_{N-4}^{t-1}+\frac{1}{20} z_{N-1}^{t-4}+\frac{37}{80} z_{N+4}^{t}+\frac{23}{80} z_{N}^{t}+\frac{7}{5}\left(z_{N}^{t}\right)^{a}+\frac{7}{5}\left(z_{N}^{t}\right)^{b}\right) \\
& =\epsilon^{l a}\left(\frac{7}{5} \tilde{u}_{s}-\frac{193}{40} \tilde{u}_{2 x}-\frac{7}{5} \tilde{u}^{a}-\frac{7}{5} \epsilon^{2} \tilde{u}^{b}\right)+\epsilon^{l+3} \text { Higher terms } .
\end{aligned}
$$

$f$ and $g$ above are mutually tropically equivalent.

For the corresponding ( $\max ,+$ )-function to $f$, the Lipschitz constant is $b>1$, and the number of the components are bounded roughly by $3 \times 10^{4}$. The error constant is roughly bounded by $10^{2}$. $k=4, D=2$ and $L=4$.

Suppose $\tilde{u}$ satisfies the equation $\frac{7}{5} \tilde{u}_{s}-\frac{193}{40} \tilde{u}_{2 x}-\frac{7}{5} \tilde{u}^{a}-\frac{7}{5} \delta \tilde{u}^{b}=0$, and admits bounded variation constants $V(\tilde{u}) \leq \tilde{V}$.

Now let us put $M_{\mu}=\max \left(2 \times 10^{3} c_{\mu}^{2}, 3 \times 10^{4}\right)$. Then by corollary 3.4 and lemma 3.2, one finds the asymptotic estimates for any $0<\epsilon \leq(200 \tilde{V})^{-1}$ :

$$
\left(\frac{\tilde{u}(x, s)}{v(s)}\right)^{ \pm 1} \leq\left(2 M_{\mu}\right)^{8 \frac{b^{\epsilon^{-2}(x+4 s)+1}-1}{b-1}}\left([\tilde{u}: v]_{5 \epsilon}\right)^{b^{\epsilon^{-2}(x+4 s)+4}} .
$$

The rest of the proof is the same as theorem 4.3 just by changing the variable $x$ as $\tilde{u}(x, s) \equiv u(p x, s)$, where $p=\sqrt{\frac{40}{193} \times \frac{7}{5}}$. Then for the variation 
constants $V(u) \leq V_{0}$ for $u$ and for any $0<\epsilon \leq\left(200 V_{0}\right)^{-1}$, the estimates hold:

$$
\left(\frac{u(x, s)}{v(s)}\right)^{ \pm 1} \leq\left(2 M_{\mu}\right)^{8 \frac{b^{\epsilon^{-2}(2 x+4 s)+1}-1}{b-1}}\left([u: v]_{5 \epsilon}\right)^{b^{\epsilon^{-2}(2 x+4 s)+4}} .
$$

This completes the proof.

\section{References}

[F] H.Fujita, On the blowing up of solutions of the Cauchy problem for $u_{t}=\Delta u+u^{1+\alpha}$, J. Fac. Sci. Univ. Tokyo (I) 13 pp. 109-124 (1966).

[H] R.Hinota, Nonlinear partial differential equations I, III, Journal of Phys. Soc. Japan 43 pp. 2074-2078, pp.2079-2086 (1977).

[K1] T.KAto, Pattern formation from projectively dynamical systems and iterations by families of maps, To appear in the Proceedings of the 1st MSJ-SI, Probabilistic Approach to Geometry.

[K2] T.Kato, Deformations of real rational dynamics in tropical geometry, Geometric and Functional Analysis 19 No 3, pp 883-901 (2009).

[KT] T.Kato And S.Tsujimoto, A rough analytic relation on partial differential equations, arXiv:1004.4981v1 math.MG (2010)

[LN] T.-Y.LEE AND W.-M.NI, Global existence, large time behaviour and life span of solutions of a semilinear parabolic Cauchy problem, Trans. Amer. Math. Soc. 333 pp. 365-378 (1992).

[LiM] G.Litvinov And V.Maslov, The correspondence principle for idempotent calculus and some computer applications, Idempotency, Ed. J.Gunawardena, Cambridge Univ. Press, pp420-443 (1998).

[Lo] J.Logan, An introduction to nonlinear partial differential equations, John Wiley and Sons Inc. Publ., (2008).

[Mi] G.Mikhalkin, Amoebas and tropical geoemtry, in Different faces of geometry eds, S.Donaldson, Y.Eliashberg and M.Gromov, Kluwer academic plenum publ., (2004). 
[V] O.VIro, Dequantization of real algebraic geometry on logarithmic paper, Proc. of the European Congrress of Math., (2000).

E-mail: tkato@math.kyoto-u.ac.jp

Department of Mathematics, Kyoto University,

Kyoto 606-8502, Japan 